\title{
Tidally disrupted dusty clumps as the origin of broad emission lines in active galactic nuclei
}

\author{
Jian-Min Wang ${ }^{1,2,3}, \mathrm{Pu} \mathrm{Du}^{1}$, Michael S. Brotherton ${ }^{4}$, Chen $\mathrm{Hu}^{1}$, Yu-Yang Songsheng ${ }^{1}$, \\ Yan-Rong $\mathrm{Li}^{1}$, Yong $\mathrm{Shi}^{5}$ and Zhi-Xiang Zhang ${ }^{1}$
}

${ }^{1}$ Key Laboratory for Particle Astrophysics, Institute of High Energy Physics, Chinese Academy of Sciences, 19B Yuquan Road, Beijing 100049, China

${ }^{2}$ School of Astronomy and Space Science, School of Physical Sciences, University of Chinese Academy of Sciences, 19A Yuquan Road, Beijing 100049, China

${ }^{3}$ National Astronomical Observatories of China, Chinese Academy of Sciences, 20A Datun Road, Beijing 100020, China

${ }^{4}$ Department of Physics and Astronomy, University of Wyoming, Laramie, WY 82071, USA

${ }^{5}$ School of Astronomy and Space Science, Nanjing University, Nanjing 210093, China

Type 1 active galactic nuclei display broad emission lines, regarded as arising from photoionized gas moving in the gravitational potential of a supermassive black hole ${ }^{12}$. The origin of this broad-line region gas is unresolved so far ${ }^{1 / 2}$, however. Another component is the dusty torus $^{4}$ beyond the broad-line region, likely an assembly of discrete clumps ${ }^{5 / 6}$, 7 that can hide the region from some viewing angles and make them observationally appear as Type 2 objects. Here we report that these clumps moving within the dust sublimation radius, like the molecular cloud $\mathrm{G} 2$ discovered in the Galactic center $\stackrel{8}{\text {, }}$, will be tidally disrupted by the hole, resulting in some gas becoming bound at smaller radii while other gas is ejected and returns 
to the torus. The clumps fulfill necessary conditions to be photoionized $\sqrt[9]{ }$. Specific dynamical components of tidally disrupted clumps include spiral-in gas as inflow, circularized gas, and ejecta as outflow. We calculate various profiles of emission lines from these clouds, and find they generally agree with $\mathrm{H} \beta$ profiles of Palomar-Green quasars ${ }^{10}$. We find that asymmetry, shape and shift of the profiles strongly depend on [O III] luminosity, which we interpret as a proxy of dusty torus angles. Tidally disrupted clumps from the torus may represent the source of the broad-line region gas.

The mass of individual clumps can be estimated by the tidal disruption condition at the dust sublimation radius given by the inner edge of torus, $D_{\text {sub }} \approx 0.4 L_{45}^{1 / 2} T_{1500}^{-2.6} \mathrm{pc}$, where $L_{45}=$ $L_{\mathrm{UV}} / 10^{45} \mathrm{erg} \mathrm{s}^{-1}$ and $T_{1500}=T_{\mathrm{sub}} / 1500 \mathrm{~K}$ is the dust sublimation temperature ${ }^{11}$ [6]12] The tidal disruption happens at or inside the Roche limit of $D_{\text {tid }}=\left(M_{\bullet} / M_{\mathrm{C}}\right)^{1 / 3} R_{\mathrm{C}}$, where $M_{\bullet}$ is the black hole mass, $M_{\mathrm{C}}$ is the clump mass, and $R_{\mathrm{C}}=\left(M_{\mathrm{C}} / \pi N_{\mathrm{H}} m_{\mathrm{p}}\right)^{1 / 2}$ is the size, where $N_{\mathrm{H}}$ is the column density and $m_{\mathrm{p}}$ is the proton mass. Dust-free clouds are disrupted when $D_{\text {tid }} \lesssim D_{\text {sub }}$, and therefore the largest dust-free clouds are given by $M_{\mathrm{C}} / M_{\oplus} \approx 2.4 \epsilon^{3} M_{8} T_{1500}^{-15.6} N_{24}^{3}$, their size is $R_{\mathrm{C}}=5.1 \times 10^{13} \epsilon^{3 / 2} M_{8}^{1 / 2} N_{24} T_{1500}^{-7.8} \mathrm{~cm}$ and density $n_{\mathrm{C}}=1.5 \times 10^{10} \epsilon^{-3 / 2} M_{8}^{-1 / 2} T_{1500}^{7.8} \mathrm{~cm}^{-3}$, where $M_{\oplus}$ is the earth mass, $\epsilon=L_{45} / M_{8}, M_{8}=M_{\bullet} / 10^{8} M_{\odot}$ and $N_{24}=N_{\mathrm{H}} / 10^{24} \mathrm{~cm}^{-2}$. For active galactic nuclei (AGNs) with $M_{\bullet} / M_{\odot}=10^{6} \sim 10^{9}$ and $\epsilon=1$, we have $M_{\mathrm{C}} / M_{\oplus} \approx 0.02 \sim$ 24.0. This estimate shows that the typical properties of the captured clumps generally fulfill the photoionization condition for broad emission lines of AGNs and quasars 9 . Moreover, we realize that $\left(M_{\mathrm{C}}, R_{\mathrm{C}}, n_{\mathrm{C}}\right)$ are very sensitive to $T_{\mathrm{sub}}$, implying that properties of broad-line regions (BLR) generally depend on processes of dust production in galactic nuclei. Note that $\epsilon=1$ corresponds 
to Eddington ratios of $L_{\mathrm{Bol}} / L_{\mathrm{Edd}} \approx 0.35$, where $L_{\mathrm{Bol}} \approx 5 L_{\mathrm{UV}}$ is used ${ }^{13}$. In the Galactic center, G2 with a mass of $3 M_{\oplus}$ captured by the central black hole ${ }^{8}$ is well in this range of $M_{\mathrm{C}}$, lending support for the idea that such captures could be common in other galactic centers.

Collisions among clumps drive them to fall within $D_{\text {sub }}$ and determine tidal capture rates. For a simple model ${ }^{14}$, the rates are about $\dot{M} \sim 1.0 M_{\odot} \mathrm{yr}^{-1}$ for typical torus parameters, which is a typical AGN accretion rate, implying a promising fueling mechanism. The stationary numbers of the captured clumps can be evaluated by continuity from torus to the BLR. The number density of captured clouds $\left(N_{\mathrm{C}}\right)$ can be simply estimated from the fuelling rates, which is written as $\dot{M} \approx 2 \Delta \Omega D_{\text {sub }}^{2} N_{\mathrm{C}} M_{\mathrm{C}} f_{v} V_{\mathrm{ff}}$, where $\Delta \Omega=2 \pi \sin \Theta_{\text {torus }}$ is the half solid angle subtended by the torus (see Figure 1), $V_{\mathrm{ff}}=\left(G M_{\bullet} / D_{\mathrm{sub}}\right)^{1 / 2}$ is the free-fall velocity and $f_{v}$ is a fraction of the free-fall velocity. The total number of clouds is about $N_{\text {tot }}=2 \Delta \Omega D_{\text {sub }}^{3} N_{\mathrm{C}} / 3$, leading to $N_{\text {tot }} \approx 6 \times 10^{7} \varepsilon_{0} f_{0.3}^{-1} M_{8}^{1 / 4} N_{24}^{-3} T_{1500}^{11.7}$, where $\varepsilon_{0}=\epsilon^{-9 / 4} \dot{M}_{1} M_{8}^{-1}, f_{0.3}=f_{v} / 0.3$ and $\dot{M}_{1}=$ $\dot{M} / 1 M_{\odot} \mathrm{yr}^{-1}$ supplied typically by the torus ${ }^{14}$. For typical values of parameters, this number is well in agreement with $3 \times 10^{7}$ clouds in NGC 4151, the lower limit based on Keck high-resolution spectroscopic observations $s^{15}$. If $T_{\text {sub }} \sim 2000 \mathrm{~K}, N_{\text {tot }} \sim 1.7 \times 10^{9}$. In principle, tidal captures by the central black hole can generally supply enough clouds to the BLR.

Capture rates are $\dot{\mathcal{R}} \sim \dot{M} / M_{\mathrm{C}} \approx 10^{5} \dot{M}_{1} M_{\mathrm{C}, 2.4}^{-1} \mathrm{yr}^{-1}$, where $M_{\mathrm{C}, 2.4}=M_{\mathrm{C}} / 2.4 M_{\oplus}$. Such a high rate makes capture events essentially continuous to form stationary spiral inflows to the black hole. Captured clumps at the mid-plane will naturally form and fuel accretion discs. However, vertical self-gravity instabilities drive the outer regions of the discs, beyond ${ }^{\sqrt{16}} R_{\mathrm{sg}} / R_{\mathrm{g}} \approx$ 
$603\left(\alpha_{0.1} / M_{8}\right)^{2 / 9} \dot{\mathscr{M}}^{4 / 9}$, to fragment into dense clouds or stars, where $R_{\mathrm{g}}=G M_{\bullet} / c^{2}$ is the gravitational radius, $\alpha_{0.1}=\alpha_{\mathrm{SS}} / 0.1$ is the viscosity parameter, and $\dot{\mathscr{M}}=\dot{M} / \dot{M}_{\text {Edd }}$ is the dimensionless rate normalized by $\dot{M}_{\mathrm{Edd}}=0.22 M_{8} M_{\odot \mathrm{yr}^{-1}}$. Such a self-gravitating disc is composed of dense clouds and diffuse dusty gas 17 . The disc conditions allow the BLR clouds to pass through, but the optical depth of the gas, $\tau \approx 4.0(\lambda / 0.7 \mu \mathrm{m})^{-1.5} N_{22}$ for Milky Way dust 19 , is sufficient to cause line emission from the BLR clouds through the disc to experience high extinction, where $N_{22}=N_{\mathrm{H}}^{\mathrm{d}} / 10^{22} \mathrm{~cm}^{-2}$ is hydrogen column density of the disc. The captured clouds from the lower half-plane torus are obscured by the disc, resulting in asymmetries of line profiles. There may be a fraction of clumps colliding with the dense clouds of the self-gravitating disc beyond $R_{\mathrm{sg}}$, but most of them can penetrate the disc between $R_{\mathrm{BLR}} \in\left[R_{\mathrm{sg}}, D_{\mathrm{sub}}\right]$, where $R_{\mathrm{BLR}} / R_{\mathrm{g}} \approx 6.2 \times 10^{3} L_{44}^{1 / 2} M_{8}^{-1}$ is the emissivity-averaged BLR radius ${ }^{20}$ and $L_{44}$ is the $5100 \AA$ luminosity in units of $10^{44} \mathrm{erg} \mathrm{s}^{-1}$.

Unlike the tidal disruption of stars 21 , the captured clumps are undergoing a more complex process, governed additionally by other significant factors such as ram pressure, ablation due to friction, and evaporation from thermal conduction with a hot medium and drag forces of the surroundings 2 . The fates of the clumps are difficult to assess due to uncertainty as to their internal states but they are either distorted or partially disrupted. See a brief discussion on this issue given in the Methods section. However, there is evidence that cloud G2 could be connected with G123, 24, implying that the clumps may split as a result of the tidal force. If the clumps are supported by an internal magnetic field at a level of milli Gauss $\frac{14}{23}$, 24, their fates will be similar to the case of stars: the tidal torque spins them up and imparts the orbital energy to the captured clumps until they split into two parts of unequal energy, one bound and the other unbound ${ }^{21}$. A fraction of the 
orbital energy is thus channelled into the ejection of gas. The ejection fraction $\left(f_{\mathrm{C}}\right)$ of the captured clumps depends on their internal states, and we estimate this dependence in the Methods section. Figure $1 a$ shows a cartoon of the present scenario.

Generally, the bound clouds experience two phases: 1) spiral-in with an elliptical orbit and 2) circularization driven by the factors mentioned previously 22$]$. We refer to them as type A and B clouds, respectively. Here circularized clouds mean their rotation dominates over their infalling velocity. Type B clouds eventually merge into the accretion disc, otherwise, there is a pile-up of material from the tidal captures. The unbound material, type $\mathrm{C}$ clouds, are ejected. We can broadly characterize the type A, B and C clouds as infalling, rotating, and outflowing, respectively.

We now present the profiles of these tidally disrupted clumps under the frame as shown by Figure 1b. All quantities are explained in Supplementary Table 1 in the Methods section. For type A clouds, $\left(\zeta_{0}, \xi_{\mathrm{A}}, \gamma_{\mathrm{A}}, i, \Theta_{\text {torus }}\right)$ are the main determinants of their contribution to the emission-line profile. $\zeta_{0}$ determines trajectories of type A clouds and hence their velocity structure. Fixing $\left(\xi_{\mathrm{A}}, \gamma_{\mathrm{A}}, i, \Theta_{\text {torus }}\right)=\left(0.9,0,60^{\circ}, 70^{\circ}\right)$, we show the dependence of the resulting profiles on $\zeta_{0}$ in Figure $2 a$. Clouds with small $\zeta_{0}$ will fall rapidly inward, for example, ones with $\zeta_{0}=0.1$, infall dominates their dynamics. Profiles are dominated by the infalling component and generally show redshifted peaks with $\zeta_{0}$ as shown by Figure $2 a$. Decreasing $\zeta_{0}$, profiles change in two ways: 1) the red peak shifts increasingly redward and 2) asymmetry is decreased. Large $-\zeta_{0}$ clouds spiral in slowly, over many orbits, showing quasi-symmetric profiles similar to type B clouds (i.e., more components from rotation-dominated clouds). These calculations show that tidally disrupted 
clumps observationally appear as asymmetric velocity profiles with centroid shifts.

Figure $2 b$ shows the profile dependence on $\gamma_{\mathrm{A}}$ for $\left(\zeta_{0}, \xi_{\mathrm{A}}, i, \Theta_{\text {torus }},\right)=\left(1,0.9,60^{\circ}, 70^{\circ}\right)$. The number of high-speed clouds increases if $\gamma_{\mathrm{A}}$ increases, and profiles shift toward the red. Figure $2 c$ shows the dependence on $\Theta_{\text {torus }}$. Small $\Theta_{\text {torus }}$ makes the capture plane more likely to have large $i$ as viewed by observers, decreases the projected velocities of the infall and hence the red wings. Double peaks appear for cases with a geometrically thin torus (i.e., small $\Theta_{\text {torus }}$ ) and low- $i$ observers. This has important implications, namely that AGNs with large torus angles will have an excess of red emission relative to the blue. The dependence of profiles on orientation is shown by Figure $2 d$. It is clear that the more face-on system leads to narrower profiles as discovered, but asymmetries remain, with excess emission on the redshifted side of the profile. Profiles also depend on $\xi_{\mathrm{A}}$, but only linearly.

For type B clouds, the profiles, which are relatively simple compared to those from type A clouds, are mainly determined by the inner radius $\left(R_{\text {in }}\right)$ and the spatial distribution of clouds along the radius $N_{\mathrm{B}} \propto v_{\mathrm{B}}^{\gamma_{\mathrm{B}}}$ as well as $i, N_{\mathrm{B}}$ is the number of clouds per unit radius, and $\gamma_{\mathrm{B}}$ is an index. We take $r_{0}=R_{\mathrm{BLR}} / R_{\mathrm{T}}^{0}=0.62$ for the circularized radius. Figure $2 e$ and $2 f$ show the spectral dependences on $\gamma_{\mathrm{B}}$ and $i$, respectively. Given an $i$, the profiles broaden with increasing $\gamma_{\mathrm{B}}$ since more clouds are located in the inner regions. The higher the inclination $i$, the narrower the profiles. Type B clouds may contribute the majority of the total BLR emission, but they could be mixed with the type A profiles with high- $\zeta_{0}$.

Generally, type C clouds simply show blue-shifted emission lines as in Figures $2 g$ and $2 h$. 
Except for inclination, the ejection velocity and $\gamma_{\mathrm{C}}$ are the main parameters governing the profiles emitted by these clouds. The larger $\gamma_{\mathrm{C}}$, the less blue-shifted the profile because there are fewer high speed clouds than the low speed. The blue peak shifts with inclination simply due to the projection of the net velocity of the clouds. Obviously, the clouds contribute to the blue excess of the total profiles shown by Figure $2 g$ and $2 h$ for different parameters.

Emission from type $\mathrm{A}, \mathrm{B}$ and $\mathrm{C}$ clouds with different ratios and shifts generate a diverse range of BLR emission-line profiles. We took efforts to calculate profiles for a larger ranges of model parameters than that shown in Figure 2 in order to find the nature of the dependence of the profiles on each parameter. From these exercises, we in the end chose 10 as primary parameters and others as the auxiliary (the latter of which we held fixed in the fittings for all objects). We apply the present model to PG quasars to determine if it can account for the nature of the observed $\mathrm{H} \beta$ profiles (see the fitting scheme explained in the Methods).

Figure 3 shows fitting results of $\mathrm{H} \beta$ profiles in four example quasars (parameters are provided by Supplementary Table 2); a complete sample of PG quasar $\mathrm{H} \beta$ profile fittings are provided in Methods, including the distributions of fitting parameters. Supplementary Table 3 lists classifications of the resultant fittings according to the relative fluxes of type A, B, and C clouds to the total. We find that the average fraction of type $\mathrm{C}$ clouds is $\left\langle f_{\mathrm{C}}\right\rangle \approx 0.1$ and $f_{\mathrm{C}} \ll\left(f_{\mathrm{A}}, f_{\mathrm{B}}\right)$ in most objects from their distributions in the Method. This nicely agrees with the estimate of Equation (77). The fittings indicate that $\mathrm{H} \beta$ profiles of PG quasars can be generally fitted very well, showing robustness of the present model. Moreover, the fittings decompose entire $\mathrm{H} \beta$ profiles for physical 
analyses.

Defining $\mathcal{A}_{\mathrm{H} \beta}, \mathcal{S}_{\mathrm{H} \beta}$ and $\mathcal{Z}_{\mathrm{H} \beta}$ as asymmetries, shapes, and shifts of profiles in Methods, we explore their correlations with the observed [O III] $\lambda 5007$ luminosity $\left(L_{[\mathrm{OIII}]}\right)$ as a proxy of dusty torus angles25] 26] Correlations are shown in Figure 4. Comparing with correlations found in PG quasars (see Table 3 in Ref. ${ }^{10}$ ), we find that the present correlations are among the strongest. Therefore, properties of the physically decomposed components $\left(\mathcal{A}_{\mathrm{H} \beta}, \mathcal{S}_{\mathrm{H} \beta}\right.$ and $\left.\mathcal{Z}_{\mathrm{H} \beta}\right)$ strongly depend on $L_{[\mathrm{OIII}]}$, and likely torus angles, lending support for the origin of the BLR from the torus.

The possibility of discrete clouds as the BLR model, supported by observations 2 , has been suggested for many years ${ }^{29}$, but their origin and supply remain open questions ${ }^{30}$ so far. In this paper, we demonstrate one possibility of the origin of the BLR. The simplified model yields major features of observational characteristics. In the future, it is worth using numerical simulations to investigate the entire dynamical evolution of the clumps which would provide more detailed comparisons with observed profiles. In such a model, the BLR is one phase in the path of fueling the black hole, physically connecting the torus, BLR, and accretion discs to each other. A self-consistent model should consider the dynamics and thermodynamics of clouds not only for profiles, but also for low/high ionisation regions emitting different lines. Reverberation of $\mathrm{H} \beta$ line and near-infrared emissions with respect to the varying continuum in AGNs, in particular ones with asymmetric $\mathrm{H} \beta$ profiles, will provide tests of the connection between the BLR and torus components. Given the importance of broad-emission lines in measuring black hole masses, this is a compelling goal. 
1. Ho, L. C. Nuclear activity in nearby galaxies. Ann. Rev. Astron. Astrophys. 46, 475-539 (2008).

2. Gaskell, C. M. What broad emission lines tell us about how active galactic nuclei work. New Astron. Rev. 53, 140-148 (2009).

3. Korista, K. What's emitting the broad emission lines? ASP Conference Series. 162, 165-176 (1999).

4. Antonucci, R. R. J. Unified models for active galactic nuclei and quasars. Ann. Rev. Astron. Astrophys. 31, 473-521 (1993).

5. Jaffe, W., Meisenheimer, K., Röttgering, H. J. A., Leinert, Ch., Richichi, A., Chesneau, O. et al. The central dusty torus in the active nucleus of NGC 1068. Nature 429, 47-49 (2004).

6. Elitzur, M. The obscuring torus in AGN. New Ast. Rev. 50, 728-731 (2006).

7. Nenkova, M., Sirocky, M. M., Ivezić, Z., Elitzur, M. AGN dusty tori. I. Handling of clumpy media. Astrophys. J. 685, 147-159 (2008).

8. Gillessen, S., Genzel, R., Fritz, T. K., Quataert, E., Alig, C., Burkert, A. et al. A gas cloud on its way towards the supermassive black hole at the Galactic centre. Nature 481, 51-54 (2012).

9. Osterbrock, D. E. \& Ferland, G. J. Astrophysics of gaseous nebulae and active galactic nuclei. Sausalito, CA: University Science Books (2006). 
10. Boroson, T. A. \& Green, R. F. The emission-line properties of low-redshift quasi-stellar objects. Astrophys. J. Supp. 80, 109-135 (1992).

11. Barvainis, R. Hot dust and the near-infrared bump in the continuum spectra of quasars and active galactic nuclei. Astrophys. J. 320, 537-544 (1987).

12. Laor, A. \& Draine, B. T. Spectroscopic constraints on the properties of dust in active galactic nuclei. Astrophys. J. 402, 441-468 (1993).

13. Elvis, M., Wilkes, B. J., McDowell, J. C., Green, R. F., Bechtold, J., Willner, S. P. et al. Atlas of quasar energy distributions. Astrophys. J. Supp. 95, 1-68 (1994).

14. Krolik, J. H. \& Begelman, M. C. Molecular tori in Seyfert galaxies - Feeding the monster and hiding it. Astrophys. J. 329, 702-711 (1988).

15. Arav, N., Barlow, T. A., Laor, A., Sargent, W. L. W. \& Blandford, R. D. Are AGN broad emission lines formed by discrete clouds? Analysis of Keck high-resolution spectroscopy of NGC 4151. Mon. Not. R. Astron. Soc. 297, 990-998 (1998).

16. Laor, A. \& Netzer, H. Massive thin accretion discs. I - Calculated spectra. Mon. Not. R. Astron. Soc. 238, 897-916 (1989).

17. Begelman, M. C., Frank, J. \& Shlosman, I. Accretion discs and the link between an AGN and its host galaxy. NATO Advanced Science Institutes (ASI) Series C 290, 373-386 (1989).

18. Collin, S. \& Zahn, J.-P. Star formation and evolution in accretion discs around massive black holes. Astron. Astrophys. 344, 433-449 (1999). 
19. Draine, B. T. Interstellar dust grains. Ann. Rev. Astron. Astrophys. 41, 241-289 (2003).

20. Bentz, M., Denney, K. D., Grier, C. J., Barth, A. J., Peterson, B. M., Vestergaard, M. et al. The low-luminosity end of the radius-luminosity relationship for active galactic nuclei. Astrophys. J. 767, 149 (2013).

21. Rees, M. J. Tidal disruption of stars by black holes of $10^{6}-10^{8}$ solar masses in nearby galaxies. Nature 333, 523-528 (1988).

22. Burkert, A., Schartmann, M., Alig, C., Gillessen, S., Genzel, R., Fritz, T. K. et al. Physics of the galactic center cloud G2, on its way toward the supermassive black hole. Astrophys. J. 750, 58 (2012).

23. Pfuhl, O., Gillessen, S., Eisenhauer, F., Genzel, R., Plewa, P. M., Ott, T. et al. The Galactic center cloud G2 and its gas streamer. Astrophys. J. 798, 111 (2015).

24. McCourt, M., O’Leary, R. M., Madigan, A.-M., Quataert, E. Magnetized gas clouds can survive acceleration by a hot wind. Mon. Not. R. Astron. Soc. 449, 2-7 (2015).

25. Simpson, C. The luminosity dependence of the type 1 active galactic nucleus fraction. Mon. Not. R. Astron. Soc. 360, 565-572 (2005).

26. Maiolino, R., Shemmer, O., Imanishi, M., Netzer, H., Oliva, E., Lutz, D. et al. Dust covering factor, silicate emission, and star formation in luminous QSOs. Astron. Astrophys. 468, 979-992 (2007). 
27. Reyes, R., Zakamska, N. L., Strauss, M. A., Green, J., Krolik, J. H., Shen, Y. et al. Space density of optically selected type 2 quasars. Astron. J. 136, 2373-2390 (2008).

28. Risaliti, G., Elvis, M., Fabbiano, G., Baldi, A., Zezas, A., Salvati, M. Occultation measurement of the size of the X-Ray-emitting region in the active galactic nucleus of NGC 1365. Astrophys. J. 659, L111-L114 (2007).

29. Krolik, J. H., McKee, C. F. \& Tarter, C. B. Two-phase models of quasar emission line regions. Astrophys. J. 249, 422-442 (1981).

30. Mathews, W. G. Structure and stability of quasar clouds. Astrophys. J. 305, 187-203 (1986). 
Acknowledgements The authors are grateful to three anonymous referees for helpful reports improving the manuscript. Todd Boroson is thanked for sending the data of PG quasar spectra. This research is supported by National Key Program for Science and Technology Research and Development (grant 2016YFA0400701) and grants NSFC-11173023, -11133006, -11373024, -11233003 and -11473002, and by Key Research Program of Frontier Sciences, CAS, Grant QYZDJ-SSW-SLH007.

Author Contributions JMW conceived the project through presenting the idea and building up the current model. JMW and DP jointly made calculations. JMW and MSB jointly wrote the manuscript. YYS and JMW fitted $\mathrm{H} \beta$ profiles of PG quasars, $\mathrm{CH}, \mathrm{ZXZ}$ and YS measured PG quasar spectra. JMW and YRL shared many discussions on clump physics. All the authors discussed the contents of the paper.

Correspondence Correspondence and requests for materials should be addressed to Jian-Min Wang (email: wangjm@ihep.ac.cn).

Competing Interests The authors declare that they have no competing financial interests. 
$\mathbf{a}$

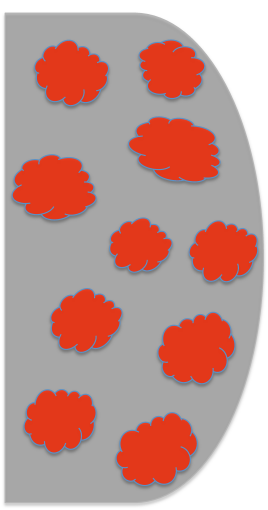

Observer

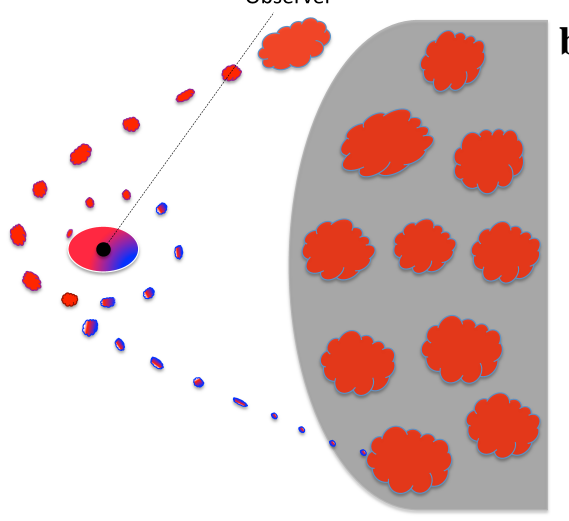

b

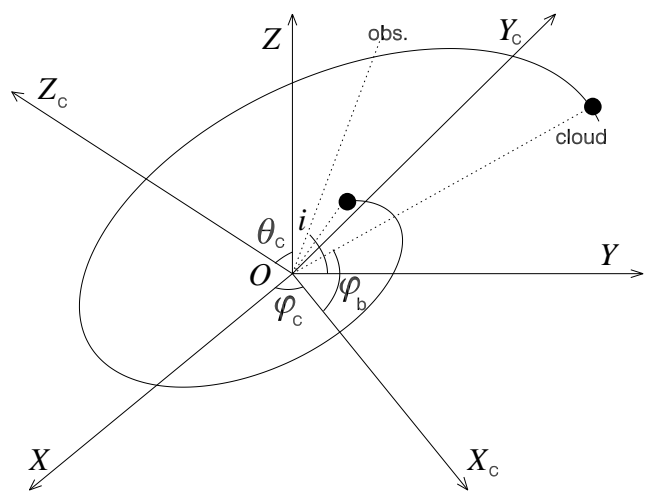

Figure 1 The scheme of the present model. Panel a shows a cartoon of the tidal disruption of clumps moving to the inner region of the torus. The red and blue colours indicate redshifts and blue shifts of line emissions after tidal disruption of clumps from the torus. Panel $\mathbf{b}$ is the frame for calculations of profiles, observers are in $Y O Z$-plane of the $X Y Z$-frame. One of the clumps is orbiting in the $X_{\mathrm{C}} O Y_{\mathrm{C}}$-plane, which is obtained by rotating the $X$-axis around the $Z$-axis $\left(\varphi_{\mathrm{C}}\right)$ and rotating the $O Z$-axis around the $O X_{\mathrm{C}}$-axis $\left(\theta_{\mathrm{C}}\right)$. The $O Y_{\mathrm{C}}$-axis is the major axis of the elliptical orbit and the $O Z_{\mathrm{C}}$-axis is the normal direction of the orbital plane. Capture planes are defined as the orbital planes of individual clumps. $\Theta_{\text {torus }}$ is the torus angle, which is defined by the angle from the mid plane to the inner edge of the torus (from $O Y$-axis to the edge in the YOZ-plane). 


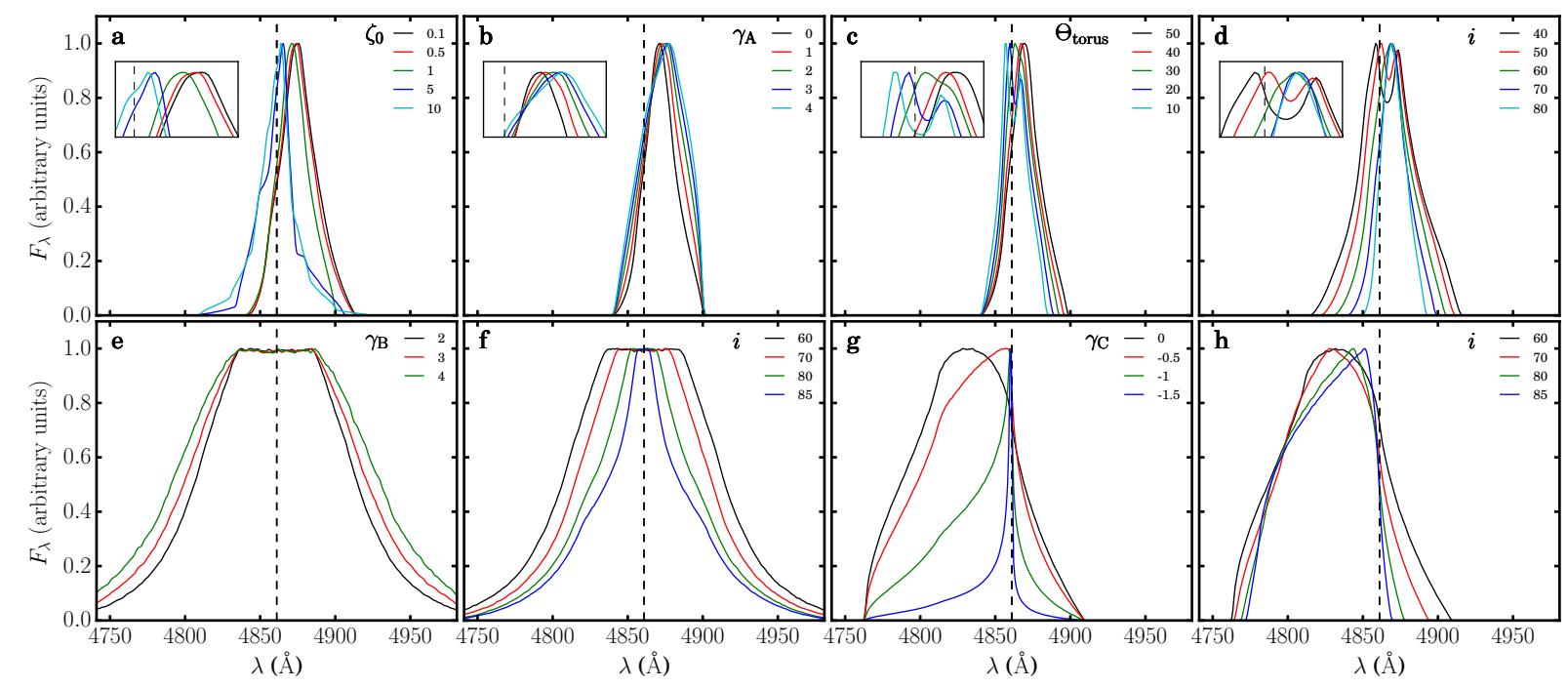

Figure 2 Various profiles of the models with a wide ranges of parameters. Panels $a-d$ show spectra for type A clouds, $e-f$ for the type B and $g$ - $h$ for the type C. The small boxes are zoom-in profiles of the peaks to clarify their fine structures. In panels $a$ - $d$, we show the profile dependence on $\left(\zeta_{0}, \gamma_{\mathrm{A}}, \Theta_{\text {torus }}, i\right)$, respectively, for given other parameters with typical values of $\left(\zeta_{0}, \gamma_{\mathrm{A}}, \Theta_{\text {torus }}, i\right)=\left(1,0,60^{\circ}, 70^{\circ}\right), \xi_{\mathrm{A}}=0.9$ and $\gamma=0$. Type B clouds have symmetric profiles, but their widths change with cloud spatial distributions and inclinations. In panel $e, \gamma_{\mathrm{B}}=(2,3,4)$ whereas dependence on $i$ and $\left(\gamma, \gamma_{\mathrm{B}}\right)=(0,2)$ is shown in panel $f$. Type $\mathrm{C}$ clouds ejected by tidal dynamics generally show profiles with blue shifts. In panel $g$, the profile dependence on $\gamma_{\mathrm{C}}$ is shown for a fixed $\left(\xi_{\mathrm{C}}, i\right)=\left(2,60^{\circ}\right)$ whereas the dependence on $i$ for a fixed $\left(\xi_{\mathrm{C}}, \gamma_{\mathrm{C}}\right)=(2,0)$ in panel $i$. The dashed lines indicate $\lambda_{0}=4861 \AA$. 


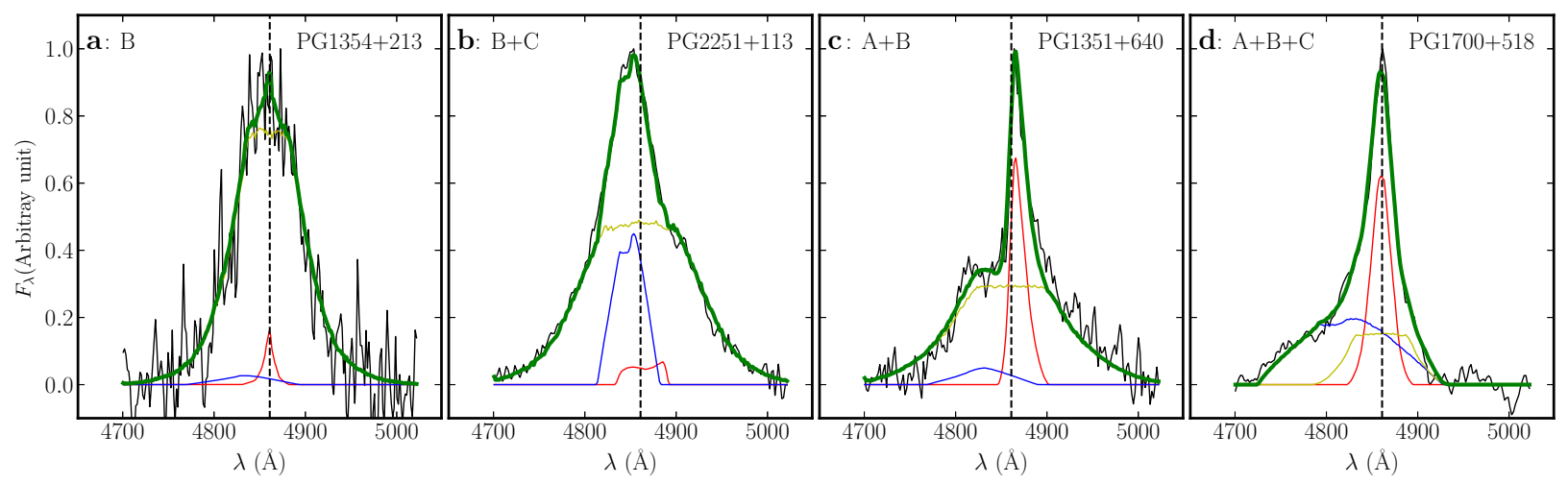

Figure 3 Best-fit models characterising the four different types of $\mathbf{H} \beta$ profiles. Object names and types are indicated in the panels. Black lines are observed spectra, the red line is from type A clouds, the yellow from type $B$, and the blue from type $C$, and green is the total. The dashed lines indicate $\lambda_{0}=4861 \AA$. We have $\chi^{2}=(1.6,2.2,2.6,3.1)$ for the best fittings of object $a-d$, respectively. The model parameters are given in Supplementary Table 2. 


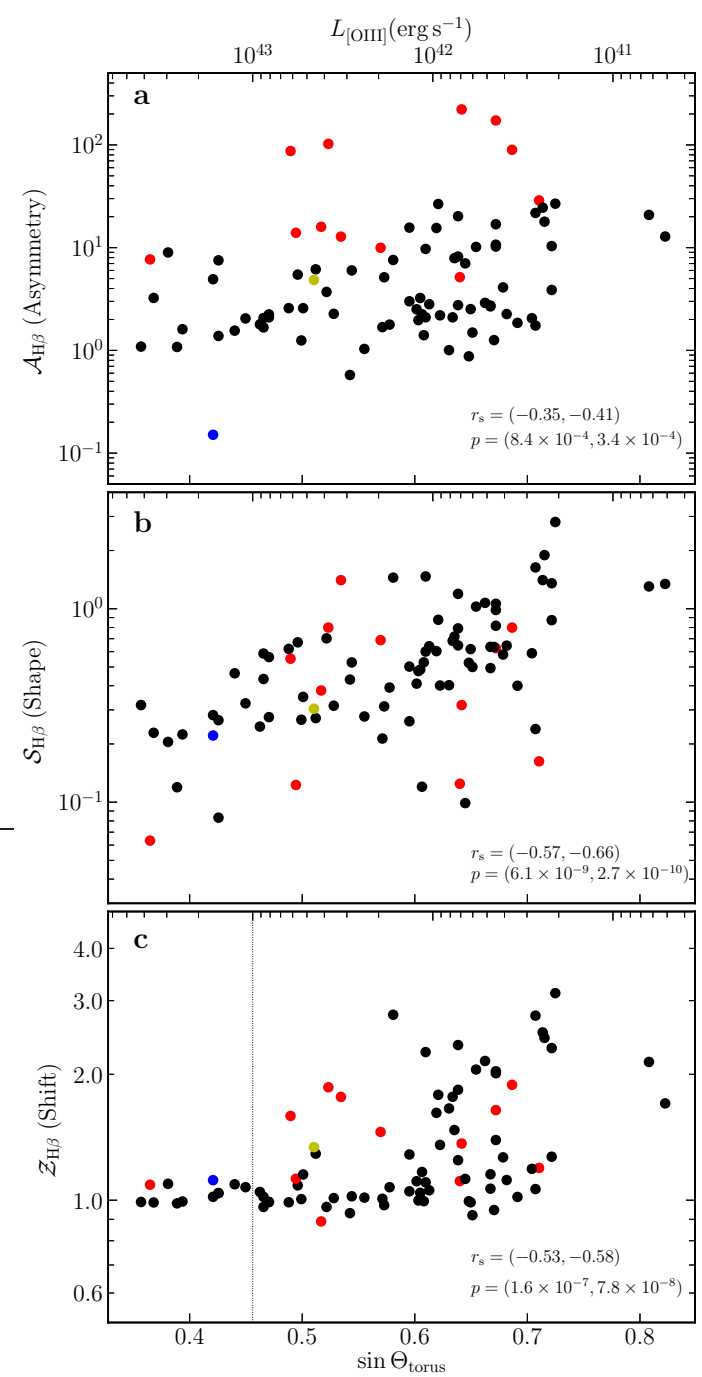

Figure 4 Correlations of asymmetries, shapes and shifts with [O III] luminosity as a proxy of torus angles (the $\Theta_{\text {torus }}-L_{\text {[OIII }]}$ relation is given by Equation 14). The correlations are among the most significant in $P G$ quasars ${ }^{10}$. The red points are $(A+B)$ objects, the one blue point and one yellow are $(B+C)$ and $B$ objects, respectively. Spearman correlation coefficients and null-probabilities are indicated on the panels (the first values are for the entire sample and the second for all black points). The dependence of $\mathcal{A}_{\mathrm{H} \beta}$, $\mathcal{S}_{\mathrm{H} \beta}$ and $\mathcal{Z}_{\mathrm{H} \beta}$ on $\Theta_{\text {torus }}$ indicates the origin of the BLR from torus. Spectral shifts increase only in quasars with $L_{[\mathrm{OIII}]} \lesssim 10^{43} \mathrm{erg} \mathrm{s}^{-1}$ (the dotted line). Average error bars of $\mathcal{A}_{\mathrm{H} \beta}, \mathcal{S}_{\mathrm{H} \beta}$ and $\mathcal{Z}_{\mathrm{H} \beta}$ are discussed in the Methods. 


\section{Methods}

Comparison with the Galactic center Recently the black hole at the Galactic center was observed to capture the molecular cloud G2, massing $\sim 3 M_{\oplus}$, at a distance of $\sim 2400$ Schwartzschild radii $\sqrt{23}$. The tidal interaction of the black hole and G2 produced a number of observational features 23 . As we showed for typical values of parameters, the range of captured mass nicely spans the properties of the G2 cloud in the Galactic center. Such an event motivated us to think about other galaxies including AGNs: tidal captures of molecular clouds could be a common phenomena in galactic centers. As a reservoir of gas, the dusty torus provides a potential source of BLR gas via tidal captures, which could unite the torus, BLR, and accretion discs in AGNs.

The mass of captured clouds is sensitive to black hole mass, very sensitive to the ratio of $\epsilon$, and also sensitive in particular to the dust sublimation temperature. Our estimate is only valid for AGNs with normal Eddington ratios since the sublimation radius depends on the spectral energy distributions (SEDs) (SDSS quasars have $L_{\mathrm{Bol}} / L_{\mathrm{Edd}}=0.1 \sim 0.5$ distributed in a fairly narrow range, see Figure 12 in Ref. ${ }^{[31}$ ). Considering a wide range of black hole mass, $M_{\mathrm{C}}$ is mostly controlled by $M_{\bullet}$ and $T_{\text {sub }}$. We note that $T_{\text {sub }}$ can reach even $2000 \mathrm{~K}$ influenced by effects of gas density or on chemical composition ${ }^{12}$, leading to a vast change of $M_{\mathrm{C}}$ by a factor of 90 due to the extreme sensitivity of $M_{\mathrm{C}} \propto T_{1500}^{-15.6}$. This strong dependence implies that the BLR clouds rely on dust composition (which is related to star formation history of accretion discs $\frac{18}{32}$, circumnuclear regions, and even of bulge regions). This could explain the dependence of metallicity on black hole mass or Eddington ratio in light of sensitivity of line flux ratios to metallicity 33 34 35 . Further exploring these relations will aid our understanding of issues of galactic center evolution. We keep 
all of these as open hypotheses in mind, which are needed to explore in detail the capture of clumps through numerical simulations.

Dynamics of tidally disrupted clumps. A dusty torus is recognized today to exist in AGNs generally. There is increasing evidence that the torus is not uniform, but rather composed of randomly moving dusty clumps; for instance, interferometric mid-infrared observations of NGC 1068 show the presence of dusty clumps ${ }^{5}$. Clumpy torus models are now commonly employed to explain the near-to-mid infrared continuum in quasars and Seyfert galaxies ${ }^{7,[36}$, 37, 38, 39, 40, 41] 42, 43] 44 . The discovery of radial motion of ionised gas deduced from quasar spectra ${ }^{45}$, and in particular, the systematical shifts of the intermediate component of $\mathrm{H} \beta$ line with the same shifts of Fe II in SDSS large sample of quasars, imply a likely supply of the BLR gas from the inner edge of torus 4647 .

The tidally captured clumps are undergoing complicated processes. It has been shown that collisions among clumps inside the torus are an efficient way to lose their angular momentum, leading to an inflow to fuel the central black hole. The rate of mass inflow depends on several factors, such as: the inelasticity of collisions $\left(\delta_{\text {diss }}\right)$, the fraction of the cloud's mass which participates in the collisions $\left(f_{m}\right)$, the covering factor of the torus $(\mathscr{C})$, the dispersion velocity $(\Delta v)$, and the column density of clumps $\left(N_{\mathrm{H}, \mathrm{t}}\right)$. The rates can be written as (see Equation 15 in Ref.!14)

$$
\dot{M} \approx 1.0 R_{0.4}^{1 / 2} M_{8}^{1 / 2}\left(\frac{f_{m}}{0.2}\right)\left(\frac{\delta_{\text {diss }}}{0.3}\right)\left(\frac{\mathscr{C} N_{\mathrm{H}, \mathrm{t}}}{10^{24} \mathrm{~cm}^{-2}}\right)\left(\frac{\Delta v_{z}}{v_{\text {orb }}}\right)\left(\frac{\Delta v}{v_{\text {orb }}}\right) M_{\odot} \mathrm{yr}^{-1}
$$

where $R_{0.4}$ is the inner edge of the torus in units of 0.4 parsec, $v_{\text {orb }}$ is the orbital velocity at $R_{0.4}$, $\Delta v_{z}$ and $\Delta v$ are the dispersion velocities of clumps in the vertical and total directions, respectively. Details of the colliding clumps, such as $f_{m}$ and $\delta_{\text {diss }}$ can be found in Ref. 13. The ratios of $\Delta v / v_{\text {orb }}$ 
and $\Delta v_{z} / v_{\text {orb }}$ determine thickness of the torus. Actually, collisions of clumps provide a physical mechanism to maintain the thickness of the tori for AGN unification schemes. Given the internal physics of clumps $\left(f_{m}, \delta_{\text {diss }}\right.$ and $\left.N_{\mathrm{H}, \mathrm{t}}\right)$, on the other hand, $\mathscr{C}$ also plays a key role in the mass rate of the inflow. Equation (1) shows an evolutionary rate of mass inflow with geometry and covering factors of tori ${ }^{\sqrt{48}}$. The improved model of clumpy accretion $\operatorname{discs}^{\sqrt{49}}$ provides a similar rate of mass inflow and detailed investigations of numerical simulations are needed for torus ${ }^{50}$.

The G2 cloud has been investigated by numerical simulations that include ablation, evaporation and compression, the complex dynamics incorporating drag forces that are either linear or proportional to the square of the velocity ${ }^{23}$, and radiation pressure (see a series of equations for details in Section 4 in Ref. ${ }^{22}$ ), however, for the captured clumps in AGNs, they are undergoing variations of thermal states due to cooling and heating of the accretion disc. Several other factors can be excluded by the following considerations. The heating power generated by the selfgravity of the clumps is of order $\dot{E}_{\mathrm{sg}} \approx G M_{\mathrm{C}}^{2} \dot{R}_{\mathrm{C}} / R_{\mathrm{C}}^{2} \approx 1.4 \times 10^{28} M_{\mathrm{C}, 2.4}^{2} R_{14}^{-2} \dot{R}_{7} \mathrm{erg} \mathrm{s}^{-1}$, where $R_{14}=R_{\mathrm{C}} / 10^{14} \mathrm{~cm}$ is the typical size of the clumps, $\dot{R}_{\mathrm{C}}=d R_{\mathrm{C}} / d t$ is the contracting velocity, $\dot{R}_{7}=\dot{R}_{\mathrm{C}} / 10^{7} \mathrm{~cm} \mathrm{~s}^{-1}$. The compression power depends on the density and temperature of the surrounding medium, which usually scales as $\left(n_{\mathrm{f}}, T_{\mathrm{f}}\right) \propto R^{-1}$ in the Galactic centet ${ }^{51[52}$. Following this relation, we take $T_{\mathrm{f}}=4.1 \times 10^{7} D_{\text {sub }, 0.4}^{-1} \mathrm{~K}$ and $n_{\mathrm{f}}=3.2 \times 10^{3} D_{\text {sub }, 0.4}^{-1} \mathrm{~cm}^{-3}$, the compression power is given by $\dot{W}=4 \pi R_{c}^{2} P_{\mathrm{f}} \dot{R}_{\mathrm{C}} \approx 2.3 \times 10^{31} D_{\text {sub, } 0.4}^{-2} R_{14}^{2} \dot{R}_{7} \mathrm{erg} \mathrm{s}^{-1}$, where $P_{\mathrm{f}}=n_{\mathrm{f}} k T_{\mathrm{f}}$ is the ram pressure, $k$ is the Boltzmann constant, and $D_{\text {sub }, 0.4}=D_{\text {sub }} / 0.4 \mathrm{pc}$. The ratio of AGN heating to the compression is about $L_{\mathrm{AGN}} D_{\mathrm{sub}}^{-2} / \dot{W} R_{\mathrm{C}}^{-2} \approx 3 \times 10^{5}$ for $L_{\mathrm{AGN}} \sim 10^{45} \mathrm{erg} \mathrm{s}^{-1}$, indicating that heating fluxes of compression and self-gravity are much smaller than that of the accretion disc 
in AGNs, but it may be very important for G2 in the Galactic center. A thermal balance is then expected between the heating of AGN radiation and cooling of captured clumps.

The ablation timescale is sensitive to the density ratio of the clouds to their surroundings, which is approximately given by $t_{\mathrm{abl}} \approx 10^{8} R_{14} V_{3}^{-1}\left(n_{\mathrm{C}} / 10^{7} n_{\mathrm{f}}\right)$ yrs $=10^{8} R_{14} V_{3}^{-1} n_{10} D_{\text {sub, } 0.4}$ yrs (Equation 6 in Ref. ${ }^{[22}$ ), where $n_{10}=n_{\mathrm{C}} / 10^{10} \mathrm{~cm}^{-3}, V_{3}=V_{\mathrm{C}} / 10^{3} \mathrm{~km} \mathrm{~s}^{-1}$ is the clump velocity relative to their surroundings and $n_{\mathrm{f}}$ is used. Here we keep the assumption that clouds hold equilibrium with their surroundings. We find that $t_{\mathrm{abl}}$ is much longer than the free-fall timescale of $t_{\mathrm{ff}}=D_{\mathrm{sub}} / V_{\mathrm{ff}} \approx 375 \epsilon^{3 / 4} M_{8}^{1 / 4} T_{1500}^{-3.9} \mathrm{yrs}$. The evaporation timescale, driven by thermal conduction of the hot surrounding medium, is $t_{\text {evap }} \approx 883 \epsilon^{9 / 4} M_{8}^{-3 / 4} N_{24} T_{1500}^{-3.9}$ yrs for the saturated evaporation (Equation 64 in Ref ${ }^{53}$ ). Considering uncertainties of the parameters, we may have $t_{\text {evap }} \sim t_{\mathrm{ff}} \ll t_{\mathrm{abl}}$, indicating that the ablation can be completely neglected, but the evaporation is important, in particular, it may govern differences of high/low ionisation line regions formed during the spiral-in of the captured clumps.

A complete calculation of all the physical details of the captured clumps should invoke all of equations of the dynamics, thermodynamics, and radiation. We need to incorporate the equations listed in Ref. ${ }^{22}$ involving the cooling and heating of the accretion discs along with the volume compression driven by the ram pressure. These processes invoke thermal instability $\sqrt{54}$, leading to the rapid formation of BLR clouds (with the typical density of $\sim 10^{10-11} \mathrm{~cm}^{-3}$ ) from the tidally captured clumps during the cooling timescale of the clumps of $t_{\text {cooling }} \approx 14 T_{4}\left(n_{10} \Lambda_{23}\right)^{-1} \mathrm{~s}$, where $T_{4}=T / 10^{4} \mathrm{~K}$ and $\Lambda_{23}=\Lambda / 10^{-23} \mathrm{erg} \mathrm{s}^{-1} \mathrm{~cm}^{3}$ is the cooling functionn. Such a calculation will self-consistently generate dynamics and radiation of emission lines from the clumps (to be carried 
out in an separate paper). We also note that some clouds could be destroyed $[30$, however, the clumps are continually supplied by tidal captures from the torus. In such a way, the BLR maintains a quasi-stationary state through balancing captures and mergers with accretion discs as done for the estimation of total clouds.

There are some simplified analytical approaches to the dynamics of clouds in the BLR, such as for the case of constant mass, but they are likely insufficient $57[58] 59$. While a detailed treatment of the dynamics of a tidally captured clump is beyond the scope of the present paper, we consider how the major characteristics of the above dynamical scenario will manifest observationally. In the frame of the $X_{\mathrm{C}} Y_{\mathrm{C}} Z_{\mathrm{C}}$-plane as the tidal disruption plane shown in Figure $1 b$, for type A clouds, we describe the simplified dynamics as

$$
v_{\mathrm{A}}=v_{\mathrm{A}}^{0}\left(R / R_{\mathrm{T}}^{0}\right)^{-\alpha_{\mathrm{A}}},
$$

and angular velocity

$$
\omega_{\mathrm{A}}=\omega_{\mathrm{A}}^{0}\left(R / R_{\mathrm{T}}^{0}\right)^{-\beta_{\mathrm{A}}},
$$

for $R>R_{0}$, where $\omega_{\mathrm{A}}=d \varphi_{\mathrm{b}} / d t$ and $v_{\mathrm{A}}=-d R / d t$. We assume that tidal disruption happens at a radius of $R_{\mathrm{T}}^{0}$ and an angle of $\varphi_{0}$ with $v_{\mathrm{A}}^{0}$ and $\omega_{\mathrm{A}}^{0}$. Here $R_{0}$ is the circularized radius due to drag forces. Type A and B cloud orbits are tangent at the pericentre point.

Combining Equations (2) and (3) for $R>R_{0}$, we can write the trajectories of type A clouds

$$
r=\left[r_{\text {torus }}^{\Gamma}-\zeta_{0}^{-1} \Gamma\left(\varphi_{\mathrm{b}}-\varphi_{0}\right)\right]^{1 / \Gamma}
$$

where $r=R / R_{\mathrm{T}}^{0}, \Gamma=\left(1+\alpha_{\mathrm{A}}-\beta_{\mathrm{A}}\right), r_{\text {torus }}=R_{\text {torus }} / R_{\mathrm{T}}^{0}$ is the inner edge of the torus, and $\zeta_{0}=R_{\mathrm{T}}^{0} \omega_{\mathrm{A}}^{0} / v_{\mathrm{A}}^{0}$ mainly determining the spiral-in trajectory. Equation (4) generates various kinds 
of spiral-in trajectories and describes the clouds moving under the influence of the drag forces. The maximum $\varphi_{\mathrm{b}}$ for the spiral-in clouds is given by $\varphi_{\max }=\varphi_{0}+\zeta_{0} \Gamma^{-1}\left(r_{\text {torus }}^{\Gamma}-r_{0}^{\Gamma}\right)$, where $r_{0}=R_{0} / R_{\mathrm{T}}^{0}$. We note that $\Gamma$ is a variable, but it can not span a large range due to practical limits on $\alpha_{\mathrm{A}}$ and $\beta_{\mathrm{A}}$. The larger $\zeta_{0}$, the more cycles of the spiral-in. With Equation (4), we have velocities of

$$
\left(\begin{array}{c}
v_{X_{\mathrm{C}}}^{\mathrm{A}} \\
v_{Y_{\mathrm{C}}}^{\mathrm{A}} \\
v_{z_{\mathrm{C}}}^{\mathrm{A}}
\end{array}\right)=-v_{\mathrm{A}}^{0}\left(\begin{array}{c}
r^{-\alpha_{\mathrm{A}} \cos \varphi_{\mathrm{b}}+\zeta_{0} r^{1-\beta_{\mathrm{A}}} \sin \varphi_{\mathrm{b}}} \\
r^{-\alpha_{\mathrm{A}} \sin \varphi_{\mathrm{b}}-\zeta_{0} r^{1-\beta_{\mathrm{A}}} \cos \varphi_{\mathrm{b}}} \\
0
\end{array}\right) .
$$

and the total velocity of

$$
v_{\mathrm{A}}=v_{\mathrm{A}}^{0}\left[r^{-2 \alpha_{\mathrm{A}}}+\zeta_{0}^{2} r^{2\left(1-\beta_{\mathrm{A}}\right)}\right]^{1 / 2},
$$

in the $X_{\mathrm{C}} Y_{\mathrm{C}} Z_{\mathrm{C}}$-plane. The mass distribution of the clouds is assumed to be $N_{\mathrm{A}} \propto v_{\mathrm{A}}^{\gamma_{\mathrm{A}}}$ owing to insufficiently understood dynamics. The index $\gamma_{\mathrm{A}} \gtrsim 0$ is expected for mass conservation.

Type B clouds orbit around the black hole, but they gradually drift onto the accretion disc. For the calculation of spectral profiles, this is equivalent to a series of rings with different orientations and radii. In the $X_{\mathrm{C}} Y_{\mathrm{C}} Z_{\mathrm{C}}$-frame, we have $v_{X_{\mathrm{C}}}^{\mathrm{B}}=-v_{\mathrm{K}} \sin \varphi_{\mathrm{b}}, v_{Y_{\mathrm{C}}}^{\mathrm{B}}=v_{\mathrm{K}} \cos \varphi_{\mathrm{b}}$ and $v_{z_{\mathrm{C}}}^{\mathrm{B}}=0$, where $v_{\mathrm{K}}$ is the Keplerian velocity. For different capture events, $R$ has a distribution from $R_{0}$ to $R_{\text {in }}$, where $R_{\text {in }}$ is the inner edge of the BLR (we take $R_{\text {in }}<R_{\mathrm{BLR}}$ and assume clouds follow $\left.N_{\mathrm{B}} \propto v_{\mathrm{B}}^{\gamma_{\mathrm{B}}}\right)$. This component could contribute the majority of the observed broad emission. We note here that type A clouds observationally overlap the type B clouds if $\zeta_{0}$ is large enough.

Type C clouds also undergo interaction with their surroundings, but they escape from the black hole potential. We simplify their trajectory as a straight line after the pericentre. This tra- 
jectory is simply given by $R=R_{\mathrm{T}}^{0} / \cos \varphi_{\mathrm{b}}$, in the $X_{\mathrm{C}} Y_{\mathrm{C}} Z_{\mathrm{C}}$-frame. With insufficient details of the tidal dynamics, we assume the approximate initial velocity of the ejected clouds to be $v_{\mathrm{C}}^{0}=\xi_{\mathrm{C}} v_{\mathrm{K}}^{0}$ and $\xi_{\mathrm{C}} \gtrsim 1$, where $v_{\mathrm{K}}^{0}$ is the Keplerian velocity at $R_{\mathrm{T}}^{0}$. For the decelerating dynamics of the escaping clouds, we assume velocities in the form $v_{Y_{\mathrm{C}}}^{\mathrm{C}}=v_{\mathrm{C}}^{0}\left(R / R_{\mathrm{T}}^{0}\right)^{-\alpha_{\mathrm{C}}}, v_{X_{\mathrm{C}}}^{\mathrm{C}}=v_{z_{\mathrm{C}}}^{\mathrm{C}}=0$, and the spatial distribution is $N_{\mathrm{C}} \propto v_{\mathrm{C}}^{\gamma_{\mathrm{C}}}$, where $\alpha_{\mathrm{C}}>0$ (deceleration by drags) or $\alpha_{\mathrm{C}}<0$ (acceleration driven by radiation pressure of accretion discs), $N_{\mathrm{C}}$ is the type $\mathrm{C}$ cloud number for unit radius, and $\gamma_{\mathrm{C}}$ is the power-law index. The acceleration driven by radiation pressure depends on several factors, including column density, metallicity, and the degree of ionisation of the clumps.

Ejection fraction. The fraction of type $\mathrm{C}$ clouds depends on details of their internal states and tidal interaction. We first consider the simpler case of a solar-type star with mass of $m_{*}$ and radius of $r_{*}$, with the side extending away from the black hole having an extra velocity above orbital, whereas the side closest to the black hole has a comparable velocity deficit. This black hole tidal torque therefore forces the star to rotate until disruption, when rotational energy is comparable with self-gravitational energy. Specific energies of the stellar gas are distributed in a range of $E_{\max }=\left(G m_{*} / r_{*}\right)\left[\left(M_{\bullet} / m_{*}\right)^{1 / 3}-1\right]$ to $E_{\min }=-\left(G m_{*} / r_{*}\right)\left[\left(M_{\bullet} / m_{*}\right)^{1 / 3}+1\right]$ in a single flyby ${ }^{21}$. Taking a uniform distribution of energy over stellar mass ${ }^{21}$, confirmed by numerical simulations ${ }^{60}$, the ejection fraction can be obtained by $f_{\mathrm{C}}=E_{\max } /\left(E_{\max }-E_{\min }\right) \approx 0.5$ due to $\left(M_{\bullet} / m_{*}\right)^{1 / 3} \gg 1$. This estimate is valid only for solar-type stars since the dynamical timescale for a captured star holds $t_{\mathrm{dyn}} \ll t_{\text {orb }}$, where $t_{\text {orb }}$ is the orbital timescale. However, it does not directly apply to the clumps we consider. If $t_{\mathrm{dyn}} \gg t_{\mathrm{orb}}$ holds, the clumps will be stretched into a ring. When $t_{\mathrm{dyn}} \gtrsim \eta_{0} t_{\mathrm{orb}}$ holds, where $\eta_{0}$ is a significant fraction, the clumps will be partially ejected. 
It is difficult to derive expression of $f_{\mathrm{C}}$ without details of the internet states of the clumps, but we can derive its dependence on $\left(\epsilon, M_{\bullet}, T_{\text {sub }}\right)$ following the above arguments. The mass of the tidally captured clumps governed by the sound wave, and is given by $M_{\mathrm{C}}^{\prime} \approx \frac{4 \pi}{3} n_{\mathrm{C}} m_{\mathrm{p}}\left(c_{\mathrm{s}} t_{\text {orb }}\right)^{3}$ during one orbit $t_{\mathrm{orb}}$, where $c_{\mathrm{s}}$ is the sound speed. Actually, $M_{\mathrm{C}}^{\prime}$ is the effective mass as part of one clump can be tidally disrupted like a star, and half its mass ejected by the tidal force. We thus have the fraction of $f_{\mathrm{C}} \approx 0.5 M_{\mathrm{C}}^{\prime} / M_{\mathrm{C}} \propto\left(t_{\mathrm{orb}} / t_{\mathrm{dyn}}\right)^{3}$, where $t_{\mathrm{dyn}}$ is the dynamical timescale and $M_{\mathrm{C}}=4 \pi n_{\mathrm{C}} m_{\mathrm{p}} R_{\mathrm{C}}^{3} / 3$. The self-gravity free-fall timescale is $t_{\mathrm{sg}}=\left(4 \pi G n_{\mathrm{C}} m_{\mathrm{p}} / 3\right)^{-1 / 2}=$ $1.57 \times 10^{11} T_{1500}^{1 / 2} D_{\text {sub, } 0.4}$, where we use the pressure balance $n_{\mathrm{C}} T_{\mathrm{C}}=n_{\mathrm{f}} T_{\mathrm{f}} \approx 1.3 \times 10^{11} D_{\text {sub }, 0.4}^{-2}$, where $T_{\mathrm{C}}$ is the temperature of clouds. Setting $t_{\mathrm{dyn}}=t_{\mathrm{sg}}$ and $T_{\mathrm{C}} \approx T_{\mathrm{sub}}$, we have $t_{\mathrm{orb}} / t_{\mathrm{dyn}} \approx$ $0.48 M_{8}^{-1 / 2} D_{\text {sub }, 0.4}^{1 / 2} T_{1500}^{-1 / 2}$, yielding

$$
f_{\mathrm{C}} \approx 0.06 \epsilon^{3 / 4} M_{8}^{-3 / 4} T_{1500}^{-5.4}
$$

Holding $T_{1500}$ constant, $f_{\mathrm{C}}$ increases with $\epsilon$ and decreases with $M_{8}$. This factor $f_{\mathrm{C}}$ is determined by the competition between the self-gravity and tidal forces. Only the self-gravitating part of the clumps can be tidally disrupted and the rest is tidally captured and is not able to adjust through self-gravity and tidal forces, in order to form type A and B clouds. Since $\epsilon$ represents Eddington ratios (or accretion rates), Equation (7) shows that AGNs with high accretion rates and less massive black holes will produce more type $\mathrm{C}$ clouds, namely, more outflows. This is generally consistent with narrow line Seyfert 1 galaxies observed in UV band ${ }^{61}$, which are generally regarded as AGNs containing less massive black holes with high accretion rates. This estimate shows type $\mathrm{C}$ clouds only contribute a small fraction to the total profiles. We should keep in mind that the current estimation of $f_{\mathrm{C}}$ is obtained by a highly simplified treatment. 
Emission-line profiles. Using the rotation matrix, we transform cloud velocities in the $X_{\mathrm{C}} Y_{\mathrm{C}} Z_{\mathrm{C}}$ into the $X Y Z$-frame for the projected velocity to the line of sight

$$
\left(\begin{array}{c}
v_{\mathrm{A}}^{\|} \\
v_{\mathrm{B}}^{\|} \\
v_{\mathrm{C}}^{\|}
\end{array}\right)=\left(\begin{array}{c}
-v_{\mathrm{A}}^{0}\left(q_{1} \cos \varphi_{\mathrm{b}}+q_{2} \sin \varphi_{\mathrm{b}}\right) \\
v_{\mathrm{K}}\left(-p_{1} \sin \varphi_{\mathrm{b}}+p_{2} \cos \varphi_{\mathrm{b}}\right) \\
v_{\mathrm{C}}^{0} p_{2}\left(\cos \varphi_{\mathrm{b}}\right)^{\alpha_{\mathrm{C}}}
\end{array}\right),
$$

for type A, B and C clouds, where $q_{1}=p_{1} r^{-\alpha_{\mathrm{A}}}-p_{2} \zeta_{0} r^{1-\beta_{\mathrm{A}}}, q_{2}=p_{1} \zeta_{0} r^{1-\beta_{\mathrm{A}}}+p_{2} r^{-\alpha_{\mathrm{A}}}, p_{1}=$ $\sin \varphi_{\mathrm{C}} \cos i$ and $p_{2}=\sin \theta_{\mathrm{C}} \sin i+\cos \varphi_{\mathrm{C}} \cos \theta_{\mathrm{C}} \cos i$. For extremely high $\dot{\mathcal{R}}$, the global distribution of captured clumps has a stationary configuration. This allows us to treat the problem in a stationary way. Taking a narrow Gaussian as our emission-line profile for individual clouds, we can determine the emission from the type $\mathrm{A}, \mathrm{B}$, and $\mathrm{C}$ components for an individual tidal capture:

$$
\begin{gathered}
f_{\lambda}^{\mathrm{A}}=\int_{\varphi_{0}}^{\varphi_{\max }} N_{\mathrm{A}}\left(v_{\mathrm{A}}\right) \mathbb{G}(\lambda) d \varphi_{\mathrm{b}}, \\
f_{\lambda}^{\mathrm{B}}=\int_{r_{0}}^{r_{\mathrm{in}}} r d r \int_{0}^{2 \pi} N_{\mathrm{B}}\left(v_{\mathrm{B}}\right) \mathbb{G}(\lambda) d \varphi_{\mathrm{b}},
\end{gathered}
$$

and

$$
f_{\lambda}^{\mathrm{C}}=\int_{0}^{\pi / 2} N_{\mathrm{C}}\left(v_{\mathrm{C}}\right) \mathbb{G}(\lambda) d \varphi_{\mathrm{b}}
$$

where $\mathbb{G}(\lambda)=(\sqrt{2 \pi} \Delta \lambda)^{-1} \exp \left[-\left(\lambda-\lambda_{0}\right)^{2} / 2(\Delta \lambda)^{2}\right]$ is the Gaussian function and $\lambda_{0}$ is the intrinsic wavelength and $\Delta \lambda$ is the width of the line. Assuming high capture rates with random directions, we can write the probability distribution of orientations as proportional to $\left(\cos \theta_{\mathrm{C}}\right)^{\gamma}$, where $\gamma$ depends on the vertical structure of the torus. We now write the emission from successive captured clumps from the upper mid-plane

$$
F_{\lambda}=\int_{0}^{2 \pi} d \varphi \int_{0}^{\Theta_{\text {torus }}} d \theta\left(\cos \theta_{\mathrm{C}}\right)^{\gamma}\left(f_{\lambda}^{\mathrm{A}}+f_{\lambda}^{\mathrm{B}}+f_{\lambda}^{\mathrm{C}}\right) .
$$


The fractions from each components can be obtained from

$$
f_{\mathrm{A}}=\frac{F_{\mathrm{A}}}{F_{\text {tot }}} ; \quad f_{\mathrm{B}}=\frac{F_{\mathrm{B}}}{F_{\text {tot }}},
$$

where $f_{\mathrm{C}}=1-f_{\mathrm{A}}-f_{\mathrm{B}}, F_{\mathrm{A}, \mathrm{B}, \mathrm{C}}$ are the fluxes from type $\mathrm{A}, \mathrm{B}, \mathrm{C}$ clouds, respectively, and $F_{\text {tot }}$ is the total flux. Here we include only captures from above the mid-plane of the torus, assuming that the disc is optically thick. In Equations (9) and (12), we assume that clouds are optically thin and radiating $\mathrm{H} \beta$ photons isotropically.

Supplementary Table 1 summarises all the parameters and ranges used in our model. The first ten parameters are the primary drivers of the observed profiles whereas others are auxiliary and can be constrained by either observations or determined to reside within small ranges by physical constraints. We take $R_{\text {torus }} / R_{\mathrm{g}}=8.5 \times 10^{4} L_{45}^{1 / 2} T_{1500}^{-2.6} M_{8}^{-1}$ as the outer boundary of tidal captures consistent with the results from NIR-RM ${ }^{62}$. The circularized radius of disrupted clumps can be conveniently approximated by the reverberation mapping relation of $R_{0} \gtrsim R_{\mathrm{BLR}}$. We lack the details of specific tidal disruption locations without internal states of the clumps, but do know that $R_{\mathrm{T}}^{0} \in\left[R_{0}, R_{\text {torus }}\right]$ holds. For an illustration of the present model, we assume $R_{\mathrm{T}}^{0} / R_{\mathrm{g}}=10^{4}$ and $\varphi_{0}=\pi / 2$. Assuming the total initial velocity of $V_{\text {tot }}^{0}=\xi_{\mathrm{A}} v_{\mathrm{K}}$ at $R_{\mathrm{T}}^{0}$, we have $v_{\mathrm{A}}^{0}=\xi_{\mathrm{A}}\left(\zeta_{0}^{2}+\right.$ $1)^{-1 / 2} v_{\mathrm{K}}$ from $V_{\mathrm{tot}}^{0}=\left[\left(v_{\mathrm{A}}^{0}\right)^{2}+\left(R_{\mathrm{T}}^{0} \omega_{\mathrm{A}}^{0}\right)^{2}\right]^{1 / 2}=\left(\zeta_{0}^{2}+1\right)^{1 / 2} v_{\mathrm{A}}^{0}$, where $\xi_{\mathrm{A}}$ is a constant $\left(\xi_{\mathrm{A}}<1\right.$ for type A clouds since they are bound). Since type A clouds are bound, $\alpha_{\mathrm{A}}<1 / 2$ follows from $v_{\mathrm{A}}^{0} \leq\left(G M_{\bullet} / R_{0}\right)^{1 / 2}$ as an infall velocity less than the free-fall velocity and $\beta_{\mathrm{A}}>3 / 2$ for subKeplerian rotation. In our calculations, we take $\Gamma=-0.25\left(\alpha_{\mathrm{A}}=0.45\right.$ and $\left.\beta_{\mathrm{A}}=1.8\right), \xi_{\mathrm{A}}=0.9$ and $\gamma_{\mathrm{A}}=0$ for illustrations. For simplicity, we assume that all the captured clumps are same and tidally disrupted at the same distance to the central black hole. This approximation is actually 
equivalent to a kind of averaged captured radii for the global system of captured clumps. Future improvement of this approximation can be done by numerical simulations. We take $\lambda_{0}=4861 \AA$ and $\Delta \lambda=1 \AA$ for the $\mathrm{H} \beta$ line.

Fitting $\mathbf{H} \beta$ profiles of PG quasars We use a tested quasar spectral fitting procedure ${ }^{\sqrt{46}}$ to obtain $\mathrm{H} \beta$ profile fits between $(4761$ - 4961) $\AA$ of PG quasars after subtracting $\mathrm{H} \beta$ narrow components, Fe II, [O III] emission lines and continuum. We define $\chi^{2}=\sum_{i=1}^{N}\left(F_{\lambda_{i}}^{\text {mod }}-F_{\lambda_{i}}^{\text {obs }}\right)^{2} / \sigma_{i}^{2}$, where $F_{\lambda_{i}}^{\text {mod }}, F_{\lambda_{i}}^{\text {obs }}$ and $\sigma_{i}$ are the model, observed fluxes and errors, respectively, and $N$ is the number of wavelength points. For a given set of model parameters $\Pi$, their posterior probability distributions are given by the Bayes theorem $p(\Pi \mid \mathcal{D}) \propto \exp \left(-\chi^{2} / 2\right) p(\Pi)$, where $p(\Pi)$ is the prior distribution of model parameters. We assume it to be flat, namely, $p(\Pi)=$ a constant. The Markov Chain Monte Carlo (MCMC) method is employed to find the best fit to data $\mathcal{D}$.

As listed in Supplementary Table 1, there are 20 parameters for a full description of the global model. We attempt to optimize fitting in two aspects: 1) to reduce number of the model parameters, and 2) to reduce degeneracy among the parameters. In order to achieve the two goals, we choose the most sensitive parameters by taking into account their roles in the model. We note that emissivities should depend on radius (expressed by some auxiliary parameters of $\gamma_{A}, \gamma_{B}$ and $\left.\gamma_{\mathrm{C}}\right)$, but we only choose $\gamma_{\mathrm{A}}$ and $\gamma_{\mathrm{B}}\left(\gamma_{\mathrm{C}}\right.$ is regarded as one auxiliary because type $\mathrm{C}$ clouds are always small contribution to the total). For type A clouds, $\zeta_{0}$ is the major driver of spiral-in trajectories and infalling velocity shifts. In principle, $\xi_{\mathrm{A}}$ is independent of $\zeta_{0}$, but is degenerate with $\zeta_{0}$. We have three of $\left(\zeta_{0}, \xi_{\mathrm{A}}, \gamma_{\mathrm{A}}\right)$ for type A clouds. For type $\mathrm{B}$ clouds, $R_{0}$ (the inner and the outer radii of type $\mathrm{A}$ and $\mathrm{B}$ clouds, respectively) connects type $\mathrm{A}$ and $\mathrm{B}$ clouds, $R_{\text {in }}$ determines 
the width of the symmetric profiles, $f_{\mathrm{B}}$ does the relative flux to the total and $\gamma_{\mathrm{A}}$ determines the emissivity distributions of type $\mathrm{B}$ clouds. We have four parameters of $\left(R_{0}, R_{\mathrm{in}}, \gamma_{\mathrm{B}}, f_{\mathrm{B}}\right)$ for type $\mathrm{B}$ clouds. For type $\mathrm{C}$ clouds, $\xi_{\mathrm{C}}$ determines the shifts of blue components of spectra and $f_{\mathrm{C}}$ does the relative flux to the total. Inclination as the only external parameters of the model but still controls apparent profiles. All other parameters are listed as auxiliary ones. Therefore, the majorities of parameters controlling the profiles are $\Pi=\left(\zeta_{0}, \gamma_{\mathrm{A}}, \xi_{\mathrm{A}}, R_{0}, R_{\mathrm{in}}, \gamma_{\mathrm{B}}, f_{\mathrm{B}}, \xi_{\mathrm{C}}, f_{\mathrm{C}}, i\right)$ and the auxiliary parameters are fixed numbers given in bold fonts in Supplementary Table 1.

Torus angles play an important role in the integration of Equation (12). We used $\Theta_{\text {tours }}$ as one of the fitting parameters, however, we found that the $\left(i, \Theta_{\text {torus }}\right)$-degeneracy is too significant to yield reliable results. Actually, this is the major degeneracy in the model. Fortunately, the receding torus relation of AGN allows us to break the degeneracy. Assuming completely random orientations of AGNs, $\Theta_{\text {torus }}$ can be estimated by $f_{2}=\Delta \Omega / 2 \pi=\sin \Theta_{\text {torus }}$, where $f_{2}$ is the relative fraction of Type $2 \mathrm{AGNs}^{63}[64[65] 66$. Torus angles follow from fractions of Type 2 AGNs statistically 1

$$
\sin \Theta_{\text {torus }}=0.77-0.16 \log L_{[\mathrm{OIII}], 41},
$$

where $L_{[\mathrm{OIII}], 41}=L_{[\mathrm{OIII}]} / 10^{41} \mathrm{erg} \mathrm{s}^{-1}$, which is consistent with other empirical relation in Ref.25, 26. We set a reasonable range of $\Theta_{\text {torus }}$ in the fittings through the scatters of Equation (14). Considering the average scatters of about $\Delta f_{2} \approx 0.15$, we have $\Delta \Theta_{\text {torus }}=\Delta f_{2} / \sqrt{1-f_{2}^{2}} \approx 10^{\circ}$, where $\left\langle f_{2}\right\rangle \approx 0.6$, as the average range of $\Theta_{\text {torus }}$ for all objects.

Inclinations are difficult to estimate, but several efforts ${ }^{67}$ for quasars and Seyfert 1 galaxies show $i=40^{\circ} \sim 90^{\circ}$ (orientations of PG quasars have not been systematically investigated). In our 
MCMC implementation, we include these physical constraints so as to alleviate some degeneracies of the П-parameters. Moreover, we set a constraint of $i \geq \Theta_{\text {torus }}$, which is a necessary condition for PG quasars as type 1 AGNs. We note that both $i$ and $R_{\text {in }}$ have influences on profile widths and $f_{\mathrm{B}}$ determines its relative contribution to the total, leading to a degeneracy of $i$ with $f_{\mathrm{B}}$ and $R_{\mathrm{in}}$ in the fittings. This degeneracy can be alleviated by a self-consistent model discussed below.

Actually, it turns out that some of the П-parameters are not critical (at least for individual objects) as shown by the resultant fittings. We would like to point out that the main goal of the PG quasar profile fitting exercise is to show the model characteristics by a comparison with data, rather than the detailed fitting fine structure of the profiles of any individual objects. This allows us to obtain the major statistical comparisons of the model with data. This can be done only after we build up a self-consistent model briefly discussed in "Implications of the present model", in which numbers of some characterized parameters can be analysed.

All the fitted spectra are shown in Supplementary Figure 1 to illustrate the models for each of the quasars, with a few exceptions (see notes on them). Contributions of three cloud types to the total can be found by red, blue and yellow lines in the figure. The parameter $\Pi$-distributions are given in Supplementary Figure 2, showing that the parameters obtained by the fittings are reasonable. We attempt to find potential relationships among the fitting parameters, but only find rather weak correlations. This indicates that the parameters likely have only weak degeneracies and thus the present options of fitting parameters are quite good. 
$\mathbf{H} \beta$ profiles in PG quasars With the decomposition of $\mathrm{H} \beta$ profiles, we define the barycentre wavelength and flux as $\bar{\lambda}=\int \lambda f_{\lambda} d \lambda / \int f_{\lambda} d \lambda$ and $h=\frac{1}{2} \int f_{\lambda}^{2} d \lambda / \int f_{\lambda} d \lambda$ for the component $f_{\lambda}$, respectively. Supplementary Figure 3 illustrates a cartoon of the decomposed profiles for $(h, \bar{\lambda})$ as well as for the following three parameters. As a first-order approximation, the two parameters of $(h, \bar{\lambda})$ are complete to describe the profiles of each decomposed components (A and C). Asymmetries are mainly described by ratios of $h$ whereas shapes and shifts by $\Delta \lambda$.

Considering that component $\mathrm{B}$ is symmetric, we define the following parameters to characterize asymmetry, shape and shift of profiles

$$
\mathcal{A}_{\mathrm{H} \beta}=\frac{h_{\mathrm{A}}}{h_{\mathrm{C}}}, \quad \mathcal{S}_{\mathrm{H} \beta}=\frac{\Delta \lambda_{\mathrm{C}}+\Delta \lambda_{\mathrm{A}}}{\mathrm{FWHM}}, \quad \text { and } \quad \mathcal{Z}_{\mathrm{H} \beta}=1+\frac{\Delta \lambda_{\mathrm{C}}-\Delta \lambda_{\mathrm{A}}}{\mathrm{FWHM}}
$$

where $\Delta \lambda_{\mathrm{A}}=\bar{\lambda}_{\mathrm{A}}-\lambda_{0}, \Delta \lambda_{\mathrm{C}}=\lambda_{0}-\bar{\lambda}_{\mathrm{C}}, \lambda_{0}=4861 \AA$ and FWHM is full-width-half-maximum of the sum of broad components of the spectra. FWHM is a good indicator of line shape for Gaussian profiles, but not enough to describe general profiles. The physical meaning of the $\mathcal{S}_{\mathrm{H} \beta}$ parameter indicates the relative width of one line with respect to its FWHM, representing the shapes of profiles. In Supplementary Figure 3, panel $b$ - $d$ show shapes with $\mathcal{S}_{\mathrm{H} \beta}$. The parameter $\mathcal{Z}_{\mathrm{H} \beta}$ describes the velocity differences between the type $\mathrm{A}$ and $\mathrm{C}$ clouds. The Boroson and Green definitions ${ }^{10}$ of similar parameters are based on observed total profiles, but they are insufficiently physically understood. However, the present $\mathcal{A}_{\mathrm{H} \beta}, \mathcal{S}_{\mathrm{H} \beta}$ and $\mathcal{Z}_{\mathrm{H} \beta}$ are defined by components decomposed by the model, making it easy to explore the nature of profiles. We note that the shape and the shift defined by Ref ${ }^{10}$ don't correlate with $L_{[\mathrm{OIII}]}$ (see their Table 3 ), but only the asymmetry. We find the present asymmetry correlates with ones defined by Ref. 10 . 
We can introduce $f_{\mathrm{A}}$ and $f_{\mathrm{C}}$ as weights for

$$
\mathcal{S}_{\mathrm{H} \beta}^{\prime}=\frac{f_{\mathrm{C}} \Delta \lambda_{\mathrm{C}}+f_{\mathrm{A}} \Delta \lambda_{\mathrm{A}}}{\mathrm{FWHM}}, \text { and } \mathcal{Z}_{\mathrm{H} \beta}^{\prime}=1+\frac{f_{\mathrm{C}} \Delta \lambda_{\mathrm{C}}-f_{\mathrm{A}} \Delta \lambda_{\mathrm{A}}}{\mathrm{FWHM}}
$$

which are parallel to Equation (15). It is straightforward to show that $\mathcal{Z}_{\mathrm{H} \beta}^{\prime}$ is equivalent to the definition of mean shifts of the total profile with respect to $\lambda_{0}$. From the barycenter wavelength of the total spectra given by $\bar{\lambda}=\int \lambda f_{\lambda} d \lambda / F_{\text {tot }}$, we have $\bar{\lambda}=f_{\mathrm{A}} \bar{\lambda}_{\mathrm{A}}+f_{\mathrm{C}} \bar{\lambda}_{\mathrm{C}}+f_{\mathrm{B}} \lambda_{0}=f_{\mathrm{A}} \Delta \lambda_{\mathrm{A}}-$ $f_{\mathrm{C}} \Delta \lambda_{\mathrm{C}}+\lambda_{0}$, where $\bar{\lambda}_{\mathrm{B}}=\lambda_{0}$ is used because of the symmetry of component B. Denoting $\Delta \lambda=$ $\bar{\lambda}-\lambda_{0}$, we have $\Delta \lambda=f_{\mathrm{A}} \Delta \lambda_{\mathrm{A}}-f_{\mathrm{C}} \Delta \lambda_{\mathrm{C}}$, leading to relations of $\mathcal{Z}_{\mathrm{H} \beta}^{\prime}=1-\Delta \lambda / \mathrm{FWHM}$ and $\mathcal{S}_{\mathrm{H} \beta}^{\prime}=$ $\left(2 f_{\mathrm{A}} \Delta \lambda_{\mathrm{A}}-\Delta \lambda\right) /$ FWHM. This demonstrates that $\mathcal{Z}_{\mathrm{H} \beta}^{\prime}$ is independent of decompositions, but $\mathcal{S}_{\mathrm{H} \beta}^{\prime}$ is still dependent. Actually, $\mathcal{Z}_{\mathrm{H} \beta}^{\prime}$ is different from the Boroson-Green's definition of centroid at 3/4 maximum from the rest wavelength in units of the FWHM. We plot $\left(\mathcal{S}_{\mathrm{H} \beta}^{\prime}, \mathcal{Z}_{\mathrm{H} \beta}^{\prime}\right)$ versus $L_{[\mathrm{OIII}]}$ in Supplementary Figure 4. Comparing with Figure 4, we find that the $\left(\mathcal{S}_{\mathrm{H} \beta}^{\prime}, \mathcal{Z}_{\mathrm{H} \beta}^{\prime}\right)-L_{[\mathrm{OIII}]}$ correlations get weaker, but are still consistent generally. This suggests that the decomposition of total profiles may provide more physical insight.

The error bars of $\mathcal{A}_{\mathrm{H} \beta}, \mathcal{S}_{\mathrm{H} \beta}$ and $\mathcal{Z}_{\mathrm{H} \beta}$ are mainly determined by $(\Delta f / f)_{\mathrm{A}, \mathrm{C}}$. Using the averaged values of $\langle f\rangle_{\mathrm{A}, \mathrm{C}}=(0.5,0.1)$ and $(\Delta f / f)_{\mathrm{A}, \mathrm{C}} \approx(0.2,0.6)$ from Table 1 , we have uncertainties of $\Delta \log \left(\mathcal{A}_{\mathrm{H} \beta}, \mathcal{S}_{\mathrm{H} \beta}, \mathcal{Z}_{\mathrm{H} \beta}\right) \approx(0.27,0.14,0.14)$ on average. $\left(\mathcal{S}_{\mathrm{H} \beta}^{\prime}, \mathcal{Z}_{\mathrm{H} \beta}^{\prime}\right)$ have the similar error bars. Here we use $10 \%$ as the general uncertainty of FWHM.

Inclinations of PG quasars Inclination angle is an important parameter, but extremely difficult to estimate. The average inclination is $\langle i\rangle \approx 63^{\circ}$ obtained from the model fits to the PG sample. It is challenging to compare in a meaningful way the inclinations of individual objects with inclina- 
tions estimated by other methods, but the distributions of the presently determined inclinations are consistent with those of type 1 AGNs ${ }^{67}$. Our analyses find no significant difference of BLR inclinations between radio-loud and radio-quiet quasars. This is interesting in light of the comparison of our inclinations of radio-loud PG quasars to those estimated by the self-synchrotron Compton limit in radio-loud AGNs and quasars (see Figure 7 in Ref. ${ }^{68}$ ), which are generally consistent. Moreover, the spectral slope in the $(1700-3000) \AA$ range could be an indicator of inclination in

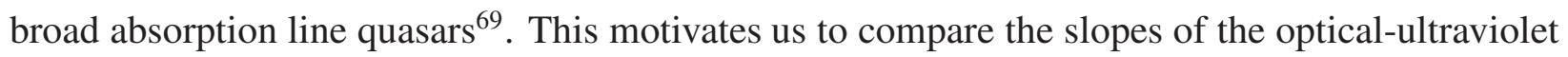
continuum of PG quasars ${ }^{2}$ with the present inclinations. Here the slope is defined by $f_{\nu} \propto \nu^{-\alpha_{\text {OUV }}}$ measured between $(1549-4861) \AA$. As shown by Supplementary Figure 5, the correlation is weak, but the trend is clear (it should be noted that $\alpha_{\text {OUV }}$ is defined in a significantly wider range than the slopes in the $(1700-3000) \AA$ range, which might weaken the correlation). This lends support to the current inclinations from the present model. Similar tests can be done for a large sample of SDSS quasars ${ }^{71}$ in the future.

Other models There are a number of ideas about the origin of the BLR gas that have been investigated. Most involve winds $52 \sqrt[73]{74}$, failed dusty winds ${ }^{75} \mid 76$ or magnetohydrodynamical (MHD)

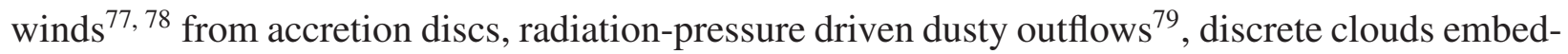
ded in a hot medium ${ }^{29}$, phenomenological models 80 81] mary, or a recent paper ${ }^{\sqrt[84]{4}}$ ), and finally condensation of warm absorbers suggested very recently Disc wind models are problematic as they fail to meet observational constraints from velocityresolved reverberation mapping of $\mathrm{AGNs}^{\sqrt{85}}$, to be inconsistent with evidence for the virialization relation of FWHM $\propto \tau_{\mathrm{H} \beta}^{-1 / 2}$ in a couple of mapped AGNs (3C 390.3, NGC 3783, NGC 5548 and 
NGC 7469 $\sqrt{\frac{86}{}}$, where $\tau_{\mathrm{H} \beta}$ is the observed lags of $\mathrm{H} \beta$ line, or suffer from predictions of small covering factors of the $\mathrm{BLR}^{87}$. MHD models are quite flexible, but difficult to observationally test. X-ray eclipses by material with BLR properties indicate that discrete clouds are present in the BLR ${ }^{28}$. Many phenomenological models involve two regions for low- and high-ionisation broad emission lines separately (see the cartoon of Figure 1 in Ref. $\left[{ }^{80}\right.$ ). The necessary conditions are extensively studied for broad emission lines: low-ionisation regions are the outer part of accretion discs, but high-ionisation line regions with a spherical geometry composed of discrete clouds whose infalling kinematics remains completely unknown (seemingly independent of accretion discs). Profiles have not been compared with observations, yet. Recently, the failed dusty wind model, which is attractive to explain the observed $R-L$ relation, receives much attention. However, much work remains to be done in order to compare this model with observations, such as the calculation of profiles of emission lines from the winds, the dependence of dynamics on vertical structure of the accretion discs. Moreover, how to form C IV regions as high-ionisation regions (depending on radiation pressure $^{76}$ somehow) which may be smaller by a factor of $5-10$ than $\mathrm{H} \beta$ regions ${ }^{88}$, fully remain open questions.

Outflows are apparently common among $\mathrm{AGNs}^{89}$, 90 , and, if they originate from the inner parts of accretion discs, there arises an interesting question: are the outflows in some way associated with the existing BLR clouds? In principle, they are spatially overlapped somehow if the outflows are approximately parallel to the disc surface, but the velocity-resolved reverberation mapping of AGNs through spectroscopically monitoring campaigns shows evidence for inflows and rotation in most $\mathrm{AGNs}^{92}$ 93 94 , and only two AGNs (NGC 3227 and Mrk 142) show evidence 
for outflows. This probably means that outflows are not the primary origin of the BLR, but also that strong interactions are not so common. However, more campaigns are needed to generally explore if there is such an interaction even if an outflow is not the primary source of the BLR.

Supposing that there is an efficient interaction between the discs winds and the BLR clouds, we would speculate above effects of interactions. First, the BLR clouds cannot be destroyed by the disc winds in the ablation timescale, but the drag force will be significantly enhanced (i.e., strongly affect $\left.\zeta_{0}\right)$. For simplicity, we estimate the wind density $n_{\mathrm{w}}=\dot{M}_{\mathrm{w}} / 4 \pi R_{\mathrm{BLR}}^{2} V_{\mathrm{w}} m_{\mathrm{p}}$, where $\dot{M}_{\mathrm{w}}$ is the wind mass rate and $V_{\mathrm{w}}$ is its velocity. Using the $R_{\mathrm{BLR}}-L$ relation 20 , we have $n_{\mathrm{w}} \approx 10^{6} \dot{M}_{0} M_{8}^{-1 / 2} L_{44}^{-3 / 4} \mathrm{~cm}^{-3}$ and thus $t_{\mathrm{abl}} \approx 2.5 \times 10^{4} R_{14} n_{10} \dot{M}_{0}^{-1} L_{44}$ yrs, where $\dot{M}_{0}=$ $\dot{M}_{\mathrm{w}} / 1 M_{\odot} \mathrm{yr}^{-1}$ and $V_{\mathrm{w}}$ is taken to be the Keperian velocity at $R_{\mathrm{BLR}}$. This is much longer than the timescales of capturing clumps. On the other hand, the thermal instability creates new clouds in the thermal times scales ${ }^{95}$. The BLR is covered by more clouds so that the observed EW of broad-lines will be enhanced significantly. Observational tests could be done by systematically comparing $\operatorname{EW}(\mathrm{H} \beta)$ of the AGNs with/without BLR outflows. Second, it has been suggested that superluminous transients might appear in AGNs if disc winds collide with the BLR clouds (the BLR clouds are treated as continuous fluid) $\sqrt{96}$. We should keep cautious about these predictions and hope future numerical simulations help understand such a complicated BLR.

Implications of the present model Given the ionisation parameter of $\Xi=L_{\mathrm{ion}} / 4 \pi R_{\mathrm{BLR}}^{2} c p_{\text {gas }}$, we have $R_{\mathrm{BLR}} \propto L_{\mathrm{ion}}^{1 / 2} \propto L_{5100}^{1 / 2}$ if photoionization dominates the physics of the BLR, where $L_{\text {ion }}$ and $L_{5100}$ are the ionizing (for hydrogen atoms) and $5100 \AA$ luminosities, respectively, and $p_{\text {gas }}$ is the gas pressure of ionized clouds in the BLR. The present model explains the $R-L$ relation in 
term of the constant ionization parameter for photoionization 20 . Why the ionisation parameter is essentially constant remains as a long-term open question ${ }^{97}$, 76 , but it is worth of investigating if it is caused by the evolution of the clumps from their capture locations until they merge with accretion discs (discussed in "Dynamics of tidally disrupted clumps"). In this paper, we emphasize that the BLR originates as a natural consequence of the tidal capture of clumps from the torus, which is consequently the source of fuel for the accretion disc. We point out that AGNs with extremely high accretion rates have $\mathrm{H} \beta$ lags much shorter than objects with the same luminosity from the $R_{\mathrm{BLR}} \propto L^{1 / 2}$ relation 98 [00. Even more complicated is the presence of multiple lags in NGC

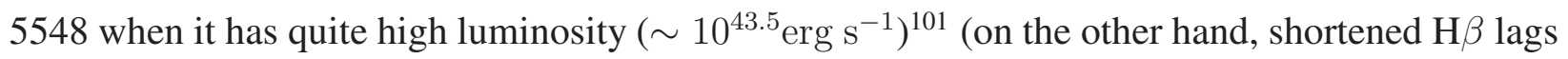
are common among AGNs with high accretion rates, but NGC 5548 only has once shown such behaviour in the last 17 monitoring campaigns, indicating that the shortening mechanisms are likely different). This at least indicates that there are sub-structures in the BLRs ${ }^{102}$. Furthermore, true type 2 AGNs lacking broad emission lines could be explained by either their central black holes having low accretion rates ${ }^{103}$, or the black holes having very high accretion rates $104[105$. The former can be easily explained by the lack of captured clumps. The later subject involves the BLR structure, which the self-shadowing effects governed by the inner discs strongly influences in AGNs with high accretion rates 100 .

In our model, tidal captures of clumps from the dusty torus determine the planes of the BLR clouds, and therefore the poloidal geometry of the BLR, namely, $\Theta_{\mathrm{BLR}} \approx \Theta_{\text {torus }}$ holds for $\mathrm{H} \beta$ regions (low-ionisation line regions), and follows torus vertical structure. If the BLR thickness can be reliably estimated by modeling reverberation mapping data $\frac{106}{107}$, an interesting correlation is 
expected between $(H / R)_{\mathrm{BLR}},(H / R)_{\text {torus }}$ of AGNs and quasars, providing one observational test of the present scenario of the BLR origin, where $H / R$ with subscripts are the relative thickness at the characterized radius of the BLR and the torus, respectively.

It is worth doing numerical simulations of the present complex processes for in-depth comparisons with observations by making a more detailed physical and self-consistent model, which includes the dynamics of interactions with surroundings (diffusive medium and even outflows developed from accretion discs), thermodynamics, and radiation. According to Equation (1), a different geometry of the torus (covering factor $\mathscr{C}$ and angle $\Theta_{\text {torus }}$ ) could lead to a different environment within $D_{\text {sub }}$ and strongly affect the subsequent evolution of captured clumps. The present model coupling a cloud's dynamics and thermodynamics, in principle, would naturally explain the existence of high/low-ionisation regions during the spiral-in to galactic centres and lead to the observed

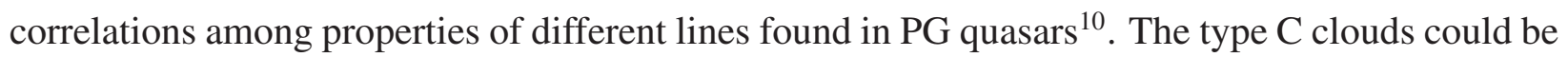
accelerated by the radiation pressure of the accretion discs and provide a potential sources of outflows, which could be related with several issues in AGNs. Accretion discs are currently presumed to power AGNs, but how to form such structures from a torus should be investigated along with mergers of BLR clouds. Observationally, future campaigns of monitoring AGNs with different $\operatorname{asymmetries}^{46] 108]}\left[109\right.$, optical Fe II $\frac{47]}{\text {, near infrared emission }} \underset{62}{ }$ and orientations deduced from the ratio of core-to-extend radio emission ${ }^{110}$ will help identify the global structure and origin of the BLR.

Data Availability Statement The data that support the plots within this paper and other findings of this study are available from the corresponding author upon reasonable request. 
31. Shen, Y., Greene, J. E., Strauss, M. A., Richards, G. T. \& Schneider, D. P. Biases in virial black hole masses: An SDSS perspective. Astrophys. J. 680, 169-190 (2008).

32. Wang, J.-M., Du, P., Baldwin, J. A., Ge, J.-Q., Hu, C. \& Ferland, G. J. Star formation in self-gravitating discs in active galactic nuclei. II. Episodic formation of broad-line regions. Astrophys. J. 746, 137 (2012).

33. Hamann, F., Korista, K. T., Ferland, G. J., Warner, C. \& Baldwin, J. Metallicities and abundance ratios from quasar broad emission lines. Astrophys. J. 564, 592-603 (2002).

34. Warner, C., Hamann, F. \& Dietrich, M. A relation between supermassive black hole mass and quasar metallicity? Astrophys. J. 596, 72-84 (2003).

35. Matsuoka, K., Nagao, T., Marconi, A., Maiolino, R. \& Taniguchi, Y. The mass-metallicity relation of SDSS quasars. Astron. Astrophys. 527, A100 (2011).

36. Nenkova, M., Ivezic, Z. \& Elitzur, M. Dust emission from active galactic nuclei. Astrophys. J. 570, L9-L12 (2002).

37. Mor, R., Netzer, H. \& Elitzur, M. Dusty structure around type-I active galactic nuclei: Clumpy torus narrow-line region and near-nucleus hot dust. Astrophys. J. 705, 298-313 (2009).

38. Mor, R. \& Netzer, H. Hot graphite dust and the infrared spectral energy distribution of active galactic nuclei. Mon. Not. R. Astron. Soc. 420, 526-541 (2012).

39. Lira, P., Videla, L, Wu, Y., Alonso-Herrero, A., Alexander, D. M. \& Ward, M. Modeling the nuclear infrared spectral energy distribution of type II active galactic nuclei. Astrophys. J. 764, 159 (2013). 
40. Netzer, H. Revisiting the unified model of active galactic nuclei. Ann. Rev. Astron. Astrophys. 53, 365-408 (2015).

41. Ichikawa, K., Packham, C., Ramos Almeida, C., Asensio Ramos, A., Alonso-Herrero, A., González-Martín, O. et al. The differences in the torus geometry between hidden and nonhidden broad line active galactic nuclei. Astrophys. J. 803, 57 (2015).

42. Fuller, L., Lopez-Rodriguez, E., Packham, C., Ramos-Almeida, C., Alonso-Herrero, A., Levenson, N. A. et al. Investigating the dusty torus of Seyfert galaxies using SOFIA/FORCAST photometry. Mon. Not. R. Astron. Soc. 462, 2618-2630 (2016).

43. Hernán-Caballero, A., Hatziminaoglou, E., Alonso-Herrero, A. \& Mateos, S. The near-to-mid infrared spectrum of quasars. Mon. Not. R. Astron. Soc. 463, 2064-2078 (2016).

44. Audibert, A., Riffel, R., Sales, D. A., Pastoriza, M. G. \& Ruschel-Dutra, D. Probing the active galactic nucleus unified model torus properties in Seyfert galaxies. Mon. Not. R. Astron. Soc. 464, 2139-2173 (2017).

45. Gaskell, C. M. A redshift difference between high and low ionization emission-line regions in QSOs - Evidence for radial motions. Astrophys. J. 263, 79-86 (1982).

46. Hu, C., Wang, J.-M., Ho, L. C., Chen, Y.-M., Bian, W.-H. \& Xue, S.-J. H $\beta$ profiles in quasars: evidence for an intermediate-line region. Astrophys. J. 683, L115-L118 (2008).

47. Hu, C., Wang, J.-M., Ho, L. C., Chen, Y.-M., Bian, W.-H. \& Xue, S.-J. A systematic analysis of Fe II emission in quasars: evidence for inflow to the central black hole. Astrophys. J. 687, $78-96(2008)$. 
48. Müller-Sánchez, F., Prieto, M. A., Mezcua, M., Davies, R. I., Malkan, M. A. \& Elitzur, M. The central molecular gas structure in LINERs with low-luminosity AGNs: evidence for gradual disappearance of the torus. Astrophys. J. 763, L1 (2013).

49. Vollmer, B. \& Beckert, T. Turbulent viscosity in clumpy accretion discs: application to the Galaxy. Astron. Astrophys. 382, 872-887 (2002).

50. Hopkins, P. F., Hayward, C. C., Narayanan, D. \& Hernquist, L. The origins of active galactic nuclei obscuration: the 'torus' as a dynamical, unstable driver of accretion. Mon. Not. R. Astron. Soc. 420, 320-339 (2012).

51. Xu, Y.-D., Narayan, R., Quataert, E., Yuan, F. \& Baganoff, F. K. Thermal X-ray iron line emission from the Galactic center black hole Sagittarius A*. Astrophys. J. 640, 319-326 (2006).

52. Schartmann, M., Burkert, A., Alig, C., Gillessen, S., Genzel, R., Eisenhauer, F. et al. Simulations of the origin and fate of the Galactic center cloud G2. Astrophys. J. 755, 155 (2012).

53. Cowie, L. L. \& McKee, C. F. The evaporation of spherical clouds in a hot gas. I - Classical and saturated mass loss rates. Astrophys. J. 211, 135-146 (1977).

54. Mathews, W. G. \& Ferland, G. What heats the hot phase in active nuclei? Astrophys. J. 323, 456-467 (1987).

55. Sutherland, R. \& Dopita, M. A. Cooling functions for low-density astrophysical plasmas. Astrophys. J. Supp. 88, 253-327 (1993). 
56. Mathews, W. G. \& Doane, J. S. Can quasar clouds form in thermal instabilities? Astrophys. J. 352, 423-432 (1990).

57. Krause, M., Burkert, A. \& Schartmann, M. Stability of cloud orbits in the broad-line region of active galactic nuclei. Mon. Not. R. Astron. Soc. 411, 550-556 (2011).

58. Plewa, P. M., Schartmann, M. \& Burkert, A. Dynamics of gas and dust clouds in active galactic nuclei. Mon. Not. R. Astron. Soc. 431, L127-L130 (2013).

59. Shadmehri, M. On the orbital motion of cold clouds in broad-line regions. Mon. Not. R. Astron. Soc. 451, 3671-3678 (2015).

60. Evans, C. R. \& Kochanek, C. S. The tidal disruption of a star by a massive black hole. Astrophys. J. 346, L13-L16 (1989).

61. Leighly, K. M. \& Moore, J. R. HST STIS ultraviolet spectral evidence of outflow in extreme narrow-line Seyfert 1 galaxies. I. Data and analysis. Astrophys. J. 611, 107-124 (2004).

62. Koshida, S., Minezaki, T., Yoshii, Y., Kobayashi, Y., Sakata, Y., Sugawara, S. et al. Reverberation measurements of the inner radius of the dust torus in 17 Seyfert Galaxies. Astrophys. J. 788, 159 (2014).

63. Osterbrock, D. E. \& Shaw, R. A. The relative number of Seyfert 2 galaxies. I - Spectra of emission-line galaxies in the Wasilewski field. Astrophys. J. 327, 89-98 (1088).

64. Tovmassian, H. M. On the relative number of Seyfert 1 and Seyfert 2 galaxies and the opening angle of dust torus. Astron. Nach. 322, 87-91 (2001). 
65. Cao, X. On the dust tori in Palomar-Green quasars. Astrophys. J. 619, 86-92 (2005).

66. Wang, J.-M., Zhang, E.-P. \& Luo, B. Evolutionary consequences of dusty tori in active galactic nuclei. Astrophys. J. 627, L5-L8 (2005).

67. Marin, F. Are there reliable methods to estimate the nuclear orientation of Seyfert galaxies? Mon. Not. R. Astron. Soc. 460, 3679-3705 (2016).

68. Ghisellini, G., Padovani, P., Celotti, A. \& Maraschi, L. Relativistic bulk motion in active galactic nuclei. Astrophys. J. 407, 65-82 (1993).

69. Baskin, A., Laor, A. \& Hamann, F. The average absorption properties of broad absorption line quasars at $800<\lambda_{\text {rest }}<3000 \AA$ and the underlying physical parameters. Mon. Not. R. Astron. Soc. 432, 1525-1543 (2013).

70. Baskin, A. \& Laor, A. What controls the C IV line profile in active galactic nuclei? Mon. Not. R. Astron. Soc. 356, 1029-1044 (2005).

71. Krawczyk, C. M., Richards, G. T., Gallagher, S. C., Leighly, K. M., Hewett, P. C., Ross, N. P. et al. Mining for dust in type 1 quasars. Astron. J. 149, 203 (2015).

72. Shlosman, I., Vitello, P. A. \& Shaviv, G. Active galactic nuclei - Internal dynamics and formation of emission clouds. Astrophys. J. 294, 96-105 (1985).

73. Murray, N., Chiang, J., Grossman, S. A. \& Voit, G. M. Accretion disc winds from active galactic nuclei. Astrophys. J. 451, 498-509 (1995). 
74. Proga, D. \& Kallman, T. R. Dynamics of line-driven disc winds in active galactic nuclei. II. Effects of disc radiation. Astrophys. J. 616, 688-695 (2004).

75. Czerny, B. \& Hryniewicz, K. The origin of the broad line region in active galactic nuclei. Astron. Astrophys. 525, L8 (2011).

76. Baskin, A., Laor, A. \& Stern, J. Radiation pressure confinement II. Application to the broadline region in active galactic nuclei. Mon. Not. R. Astron. Soc. 438, 604-619 (2014).

77. Emmering, R. T., Blandford, R. D. \& Shlosman, I. Magnetic acceleration of broad emissionline clouds in active galactic nuclei. Astrophys. J. 385, 460-477 (1992)

78. Konigl, A. \& Kartje, J. F. disc-driven hydromagnetic winds as a key ingredient of active galactic nuclei unification schemes. Astrophys. J. 434, 466-467 (1994).

79. Gaskell, C. M. \& Harrington, P. Z. Partial obscuration of innermost regions of active galactic nuclei by outflowing dusty clouds as a cause of broad-line profile and lag variability, and apparent accretion disc inhomogeneities. Mon. Not. R. Astron. Soc. arXiv:1704.06455(2017).

80. Collin-Souffrin, S., Dyson, J. E., McDowell, J. C. \& Perry, J. J. The environment of active galactic nuclei. I - A two-component broad emission line model. Mon. Not. R. Astron. Soc. 232, 539-550 (1988).

81. Xue, S.-J., Cheng, F.-Z. \& Kwan, J. Kinematic models of BLR gas and line profiles of He I $\lambda 5876$ and $\mathrm{H} \beta$ in AGNs. Science in China (Series A) 37, 487-496 (1994). 
82. Eracleous, M., Livio, M., Halpern, J. P. \& Storchi-Bergmann, T. Elliptical accretion discs in active galactic nuclei. Astrophys. J. 438, 610-622 (1995).

83. Goad, M. R., Korista, K. T. \& Ruff, A. J. The broad emission-line region: the confluence of the outer accretion disc with the inner edge of the dusty torus. Mon. Not. R. Astron. Soc. 426, 3086-3111 (2012).

84. Elvis, M. Quasar rain: the broad emission line region as condensations in the warm accretion disc wind. Astrophys. J.(in press), arXiv:1703.02956(2017).

85. Gaskell, C. M. \& Goosmann, R. W. The case for inflow of the broad-line region of active galactic nuclei. Astrophys. Space Sci. 361, 67 (2016).

86. Peterson, B. M., Peterson, B. M. 2011, in Narrow-Line Seyfert 1 Galaxies and Their Place in the Universe, eds. L. Foschini, M. Colpi, L. Gallo, et al., Proc. of Science, Trieste, Italy, NLS1, 32 (2011)

87. Murray, N. \& Chiang, J. Photoionization of disc winds. Astrophys. J. 494, 125-138 (1998).

88. Kaspi, S., Brandt, W. N., Maoz, D., Netzer, H., Schneider, D. P. \& Shemmer, O. Reverberation mapping of high-luminosity quasars: first results. Astrophys. J. 659, 997-1007 (2007).

89. Crenshaw, M., D. M., Kraemer, S. B. \& George, I. M. Mass loss from the nuclei of active galaxies. Ann. Rev. Astron. Astrophys. 41, 117-167 (2003).

90. Proga, D., Stone, J. M. \& Kallman, T. R. Dynamics of line-driven disc winds in active galactic nuclei. Astrophys. J. 543, 686-696 (2000). 
91. Tombesi, F. \& Cappi, M. On the presence of ultrafast outflows in the WAX sample of Seyfert galaxies. Mon. Not. R. Astron. Soc. 443, L104-L108 (2014).

92. Denney, K. D., Peterson, B. M., Pogge, R. W., Adair, A., Atlee, D. W., Au-Yong, K. et al. Diverse kinematic signatures from reverberation mapping of the broad-line region in AGNs. Astrophys. J. 704, L80-L84 (2009).

93. Grier, C. J., Peterson, B. M., Horne, K., Bentz, M. C., Pogge, R. W., Denney, K. D. et al. The structure of the broad-line region in active galactic nuclei. I. Reconstructed velocity-delay maps. Astrophys. J. 764, 47 (2013).

94. Du, P., Lu, K.-X., Hu, C., Qiu, J., Li, Y.-R., Huang, Y.-K. et al. Supermassive black holes with high accretion rates in active galactic nuclei. VI. Velocity-resolved reverberation mapping of the $\mathrm{H} \beta$ line. Astrophys. J. 820, 27 (2016).

95. Beltrametti, M. Thermal instabilities in radiatively driven winds - Application to emission line clouds of quasars and active galactic nuclei. Astrophys. J. 250, 18-30 (1981).

96. Moriya, T. J., Tanaka, M., Morokuma, T. \& Ohsuga, K. Superluminous transients at AGN centers from interaction between black-hole disc winds and broad-line region clouds. Astrophys. J. Letters (arXiv:1706.06855) (2017).

97. Baldwin, J., Ferland, G., Korista, K. \& Verner, D. Locally optimally emitting clouds and the origin of quasar emission lines. Astrophys. J. 455, L119-L122 (1995). 
98. Du, P., Hu, C. Lu, K.-X., Wang, F., Qiu, J., Li, Y.-R. et al. Supermassive black holes with high accretion rates in AGNs. I. First results from a new reverberation mapping campaign. Astrophys. J. 782, 45 (2014).

99. Du, P., Hu, C., Lu, K.-X., Huang, Y.-K., Cheng, C., Qiu, J. et al. Supermassive black holes with high accretion rates in AGNs. IV. $\mathrm{H} \beta$ time lags and implications for super-Eddington accretion. Astrophys. J. 806, 22 (2015).

100. Du, P., Lu, K.-X., Zhang, Z.-X., Huang, Y.-K., Wang, K., Hu, C. et al. Supermassive black holes with high accretion rates in AGNs. V. A new size-luminosity scaling relation for the BLR. Astrophys. J. 825, 126 (2016).

101. Pei, L., Fausnaugh, M. M., Barth, A. J., Peterson, B. M., Bentz, M. C., De Rosa, G. et al. Space telescope and optical reverberation mapping project. V. optical spectroscopic campaign and emission-line analysis for NGC 5548. Astrophys. J. 837, 131 (2017).

102. Li, Y.-R., Wang, J.-M. \& Bai, J.-M. A non-parametric approach to constrain the transfer function in reverberation mapping. Astrophys. J. 831, 206 (2016).

103. Nicastro, F. Broad emission line regions in active galactic nuclei: the link with the accretion power. Astrophys. J. 530, L16-L68 (1999).

104. Ho, L. C., Kim, M. \& Terashima, Y. The low-mass, highly accreting black hole associated with the active galactic nucleus 2XMM J123103.2+110648. Astrophys. J. 759, L16 (2012). 
105. Miniutti, G., Saxton, R. D., Rodrguez-Pascual, P. M., Read, A. M., Esquej, P., Colless, M. et al. A high Eddington-ratio, true Seyfert 2 galaxy candidate: implications for broad-line region models. Mon. Not. R. Astron. Soc. 433, 1764-1777 (2013).

106. Pancoast, A., Brewer, B. J. \& Treu, T. Geometric and dynamical models of reverberation mapping data. Astrophys. J. 730, 139 (2011).

107. Li, Y.-R., Wang, J.-M., Ho, L. C., Du, P. \& Bai, J.-M. A Bayesian approach to estimate the size and structure of the BLR in AGNs using RM data. Astrophys. J. 779, 110 (2013).

108. Zamfir, S., Sulentic, J. W., Marziani, P. \& Dultzin, D. et al. Detailed characterization of $\mathrm{H} \beta$ emission line profile in low- $z$ SDSS quasars. Mon. Not. R. Astron. Soc. 403, 1759-1786 (2010).

109. Runnoe, J. C., Eracleous, M., Mathes, G., Pennell, A., Boroson, T., Sigurosson, S. et al. et al. A large systematic search for close supermassive binary and rapidly recoiling black holes. II. Continued spectroscopic monitoring and optical flux variability. Astrophys. J. Supp. 221, 7 (2015).

110. Wills, B. J. \& Browne, I. W. A. Relativistic beaming and quasar emission lines. Astrophys. J. 302, 56-63 (1986). 


\section{Supplementary Table 1}

\section{A list of parameters involved in the present model}

\begin{tabular}{llcc}
\hline Parameter & \multicolumn{1}{c}{ Physical meanings } & Valid ranges & $\begin{array}{c}\text { Uncertainties } \\
(\langle\Delta X / X\rangle)\end{array}$ \\
\hline$\zeta_{0}$ & $R_{\mathrm{T}}^{0} \omega_{\mathrm{A}}^{0} / v_{\mathrm{A}}^{0}:$ determines trajectories of clumps & $0 \sim 50$ & 1.6 \\
$\gamma_{\mathrm{A}}$ & cloud A distribution index: $N_{\mathrm{A}}=N_{\mathrm{A}}^{0} v_{\mathrm{A}}^{\gamma_{\mathrm{A}}}$ & $0-4$ & 0.5 \\
$\xi_{\mathrm{A}}$ & $v_{\mathrm{A}}^{0}=\xi_{\mathrm{A}}\left(\zeta_{0}^{2}+1\right)^{-1 / 2} v_{\mathrm{K}}^{0}, v_{\mathrm{K}}^{0}$ is the Keplerian velocity at $R_{\mathrm{T}}^{0}$. & $<1$ & 0.2 \\
$R_{0} / R_{\mathrm{g}}$ & the circulaized radius of type A clouds & $\sim 6.2 \times 10^{3}$ & 0.3 \\
$R_{\mathrm{in}}$ & radius of type B clouds merging with accretion disks & $0-4$ & 0.6 \\
$\gamma_{\mathrm{B}}$ & cloud B distribution index: $N_{\mathrm{B}}=N_{\mathrm{B}}^{0} v_{\mathrm{B}}^{\gamma_{\mathrm{B}}}$ & $0 \sim 1$ & 0.5 \\
$f_{\mathrm{B}}$ & fraction of type B clouds & $>1$ & 0.3 \\
$\xi_{\mathrm{C}}$ & $v_{\mathrm{C}}^{0}=\xi_{\mathrm{C}} v_{\mathrm{K}}^{0}, v_{\mathrm{C}}^{0}$ is the ejection velocity of type C clouds at $R_{\mathrm{T}}^{0} \cdot$ & 0.3 \\
$f_{\mathrm{C}}$ & fraction of type C clouds, properties shown by Equation $(7)$ & $0 \sim 1$ & 0.6 \\
$i$ & inclination angle of observers, $i=0^{\circ}$ (edge-on) and $i=90^{\circ}$ (face-on) & $\gtrsim 40^{\circ}$ & 0.2 \\
\hline
\end{tabular}

Auxiliary Parameters (the fixed values are listed by the numbers in bold fonts)

\begin{tabular}{llc}
\hline$\Theta_{\text {torus }}$ & half angle of torus given by the $\Theta_{\text {torus }}-L_{[\mathrm{OIII}]}$ relation & Ref. $^{1}$ \\
$\alpha_{\mathrm{A}}$ & cloud A velocity index: $v_{\mathrm{A}}=v_{\mathrm{A}}^{0}\left(R / R_{\mathrm{T}}^{0}\right)^{-\alpha_{\mathrm{A}}}$ & $\mathbf{0 . 4 5} ; \leq 0.5$ \\
$\alpha_{\mathrm{C}}$ & cloud C velocity index: $v_{\mathrm{C}}=v_{\mathrm{C}}^{0}\left(R / R_{\mathrm{T}}^{0}\right)^{-\alpha_{\mathrm{C}}}$ & $\mathbf{0 . 3 0} ; \leq 0.5$ \\
$\beta_{\mathrm{A}}$ & cloud A angular velocity index: $\omega_{\mathrm{A}}=\omega_{\mathrm{A}}^{0}\left(R / R_{\mathrm{T}}^{0}\right)^{-\beta_{\mathrm{A}}}$ & $\mathbf{1 . 8} ; \geq 1.5$ \\
$\gamma_{\mathrm{C}}$ & cloud C distribution index: $N_{\mathrm{C}}=N_{\mathrm{C}}^{0} v_{\mathrm{C}}^{\gamma_{\mathrm{C}}}$ & $\mathbf{0} ; \sim 0.5$ \\
$\gamma$ & vertical distribution of clumps in torus: $\propto\left(\cos \theta_{\mathrm{C}}\right)^{\gamma}$ & $\mathbf{1} ; \sim 1$ \\
$\Gamma$ & $1+\alpha_{\mathrm{A}}-\beta_{\mathrm{A}}$ & $\mathbf{1 0 ^ { 4 }} ;\left(R_{0}, R_{\text {torus }}\right)$ \\
$R_{\mathrm{T}}^{0} / R_{\mathrm{g}}$ & tidal disruption radius of clumps. & \\
$R_{\mathrm{BLR}}$ & the emissivity-averaged radius of the BLR determined by RM. & \\
$\varphi_{0}$ & position angle of tidal event. & $\varphi_{0}=\pi / 2$ \\
$\omega_{\mathrm{A}}^{0}$ & angular velocity of type A clouds at $R_{\mathrm{T}}^{0}$. & absorbed by $\zeta_{0}$ \\
\hline
\end{tabular}

Note: the averaged $\langle\Delta X / X\rangle$ is obtained by the statistic of individual $\Delta X / X$ of the sample. Here $X$ is any one of the fitting parameters in the model. 


\section{Supplementary Table 2}

\section{Resultant parameters of best-fittings of four quasars}

\begin{tabular}{lcccc}
\hline Parameter & PG1354+213 & PG2251+113 & PG1351+640 & PG1700+518 \\
\hline$\zeta_{0}$ & $6.1_{-6}^{+10}$ & $0.71_{-0.5}^{+1.5}$ & $0.02_{-0.02}^{+5}$ & $38_{-31}^{+13}$ \\
$\xi_{\mathrm{A}}$ & $0.36_{-0.1}^{+0.3}$ & $0.59_{-0.1}^{+0.2}$ & $0.42_{-0.1}^{+0.1}$ & $0.63_{-0.1}^{+0.1}$ \\
$\gamma_{\mathrm{A}}$ & $0.79_{-0.79}^{+1.4}$ & $3.6_{-1.8}^{+0.9}$ & $0.51_{-0.2}^{+0.9}$ & $1.5_{-0.5}^{+0.9}$ \\
$R_{0}\left(10^{3} R_{\mathrm{g}}\right)$ & $3.2_{-0.9}^{+1.2}$ & $2.6_{-0.7}^{+1.0}$ & $1.4_{-0.8}^{+1.5}$ & $7.5_{-2.5}^{+1.5}$ \\
$R_{\mathrm{in}}\left(10^{3} R_{\mathrm{g}}\right)$ & $0.31_{-0.1}^{+0.2}$ & $0.38_{-0.1}^{+0.1}$ & $0.39_{-0.1}^{+0.1}$ & $3.6_{-0.9}^{+1.3}$ \\
$\gamma_{\mathrm{B}}$ & $1.2_{-0.1}^{+0.1}$ & $2.1_{-1.0}^{+0.9}$ & $2.8_{-1.8}^{+0.9}$ & $3.0_{-1.3}^{+0.9}$ \\
$f_{\mathrm{B}}$ & $0.95_{-0.6}^{+0.1}$ & $0.78_{-0.2}^{+0.1}$ & $0.73_{-0.1}^{+0.1}$ & $0.24_{-0.1}^{+0.0}$ \\
$\xi_{\mathrm{C}}$ & $2.7_{-1.1}^{+0.5}$ & $1.1_{-0.5}^{+0.2}$ & $2.2_{-0.4}^{+0.1}$ & $2.9_{-0.1}^{+0.1}$ \\
$f_{\mathrm{C}}$ & $0.02_{-0.02}^{+0.1}$ & $0.18_{-0.1}^{+0.1}$ & $0.04_{-0.01}^{+0.01}$ & $0.44_{-0.1}^{+0.1}$ \\
$i\left(^{\circ}\right)$ & $75_{-13}^{+8}$ & $66_{-9}^{+9}$ & $74_{-9}^{+5}$ & $61_{-1}^{+2}$ \\
\hline & & Line profiles & & \\
\hline $\mathcal{A}_{\mathrm{H} \beta}$ & 4.85 & 0.15 & 12.8 & 2.90 \\
$\mathcal{S}_{\mathrm{H} \beta}$ & 0.30 & 0.22 & 1.41 & 1.07 \\
$\mathcal{Z}_{\mathrm{H} \beta}$ & 1.34 & 1.12 & 1.77 & 2.15 \\
\hline $\mathrm{P}_{0}$ & & & & \\
\hline & & & & \\
\hline
\end{tabular}

Parameters of all the PG quasars will be provided on request. 


\section{Supplementary Table 3}

\section{Classifications of resultant fittings}

\begin{tabular}{|c|c|c|}
\hline Model & Number & Objects (total of 87 PG quasars) \\
\hline B & 1 & $1354+213$ \\
\hline $\mathrm{B}+\mathrm{C}$ & 1 & $2251+113$ \\
\hline \multirow[t]{2}{*}{$\mathrm{A}+\mathrm{B}$} & 12 & $0003+199,0934+013,1004+130,1012+008,1103-006,1211+143,1351+236$ \\
\hline & & $1351+640,1512+370,1534+580,1617+175,2233+134$ \\
\hline \multirow[t]{11}{*}{$\mathrm{A}+\mathrm{B}+\mathrm{C}$} & 73 & $0003+158,0007+106,0026+129,0043+039,0049+171,0050+124,0052+251$ \\
\hline & & $0157+001,0804+761,0838+770,0844+349,0921+525,0923+129,0923+201$ \\
\hline & & $0947+396,0953+414,1001+054,1011-040,1022+519,1048-090,1048+342$ \\
\hline & & $1049-006,1100+772,1114+445,1115+407,1116+215,1119+120,1121+422$ \\
\hline & & $1126-041,1149-110,1151+117,1202+281,1216+069,1226+023,1229+204$ \\
\hline & & $1244+026,1259+593,1302-102,1307+085,1309+355,1310-108,1322+659$ \\
\hline & & $1341+258,1352+183,1402+261,1404+226,1411+442,1415+451,1416-129$ \\
\hline & & $1425+267,1426+015,1427+480,1435-067,1440+356,1444+407,1448+273$ \\
\hline & & $1501+106,1519+226,1535+547,1543+489,1545+210,1552+085,1612+261$ \\
\hline & & $1613+658,1626+554,1700+518,1704+608,2112+059,2130+099,2209+184$ \\
\hline & & $2214+139,2304+042,2308+098$ \\
\hline
\end{tabular}

B: $f_{\mathrm{A}, \mathrm{C}} \leq 5 \% ; \mathrm{B}+\mathrm{C}: f_{\mathrm{A}} \leq 5 \% ; \mathrm{A}+\mathrm{B}: f_{\mathrm{C}} \leq 5 \% ; \mathrm{A}+\mathrm{B}+\mathrm{C}: f_{\mathrm{A}, \mathrm{B}, \mathrm{C}}>5 \%$.

If it is less than 5\%, we consider that component to be negligible. There are only two profiles with B and $(B+C)$ components, only 12 objects with $(A+B)$, while the majority of 73 objects employ $(A+B+C)$. Generally, type B clouds are necessary in all objects, type A appears in $85 / 87$ objects, and the type C in $74 / 87$. 


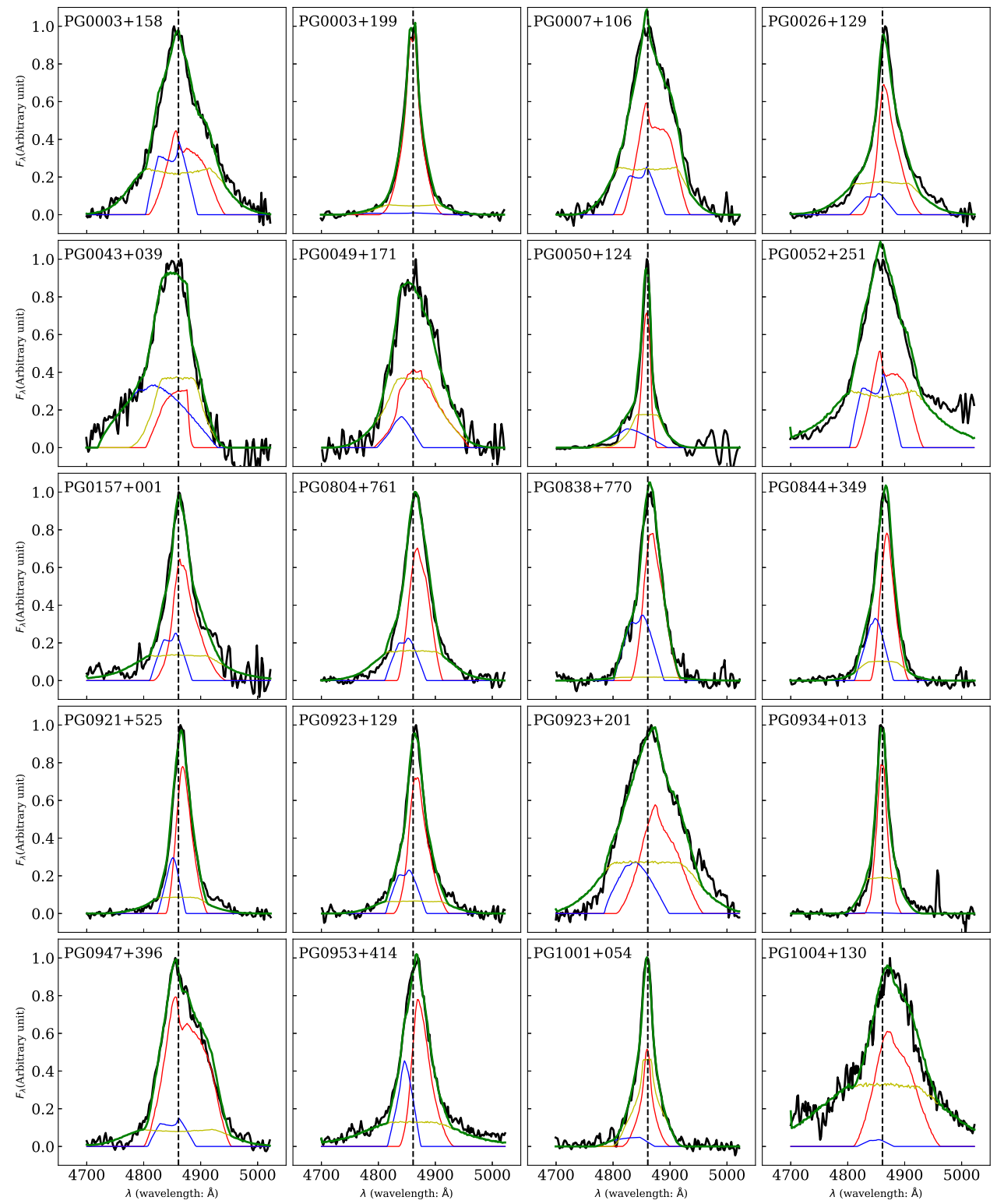

Supplementary Figure 1: Comparisons of our model fits with observed $\mathbf{H} \beta$ profiles of PG quasars. Solid black lines are the observed profiles, red lines are from type A clouds, the yellow from type B and the blue from type C and the green is the total of the three cloud types. The dashed lines are $\lambda_{0}=4861 \AA$. 


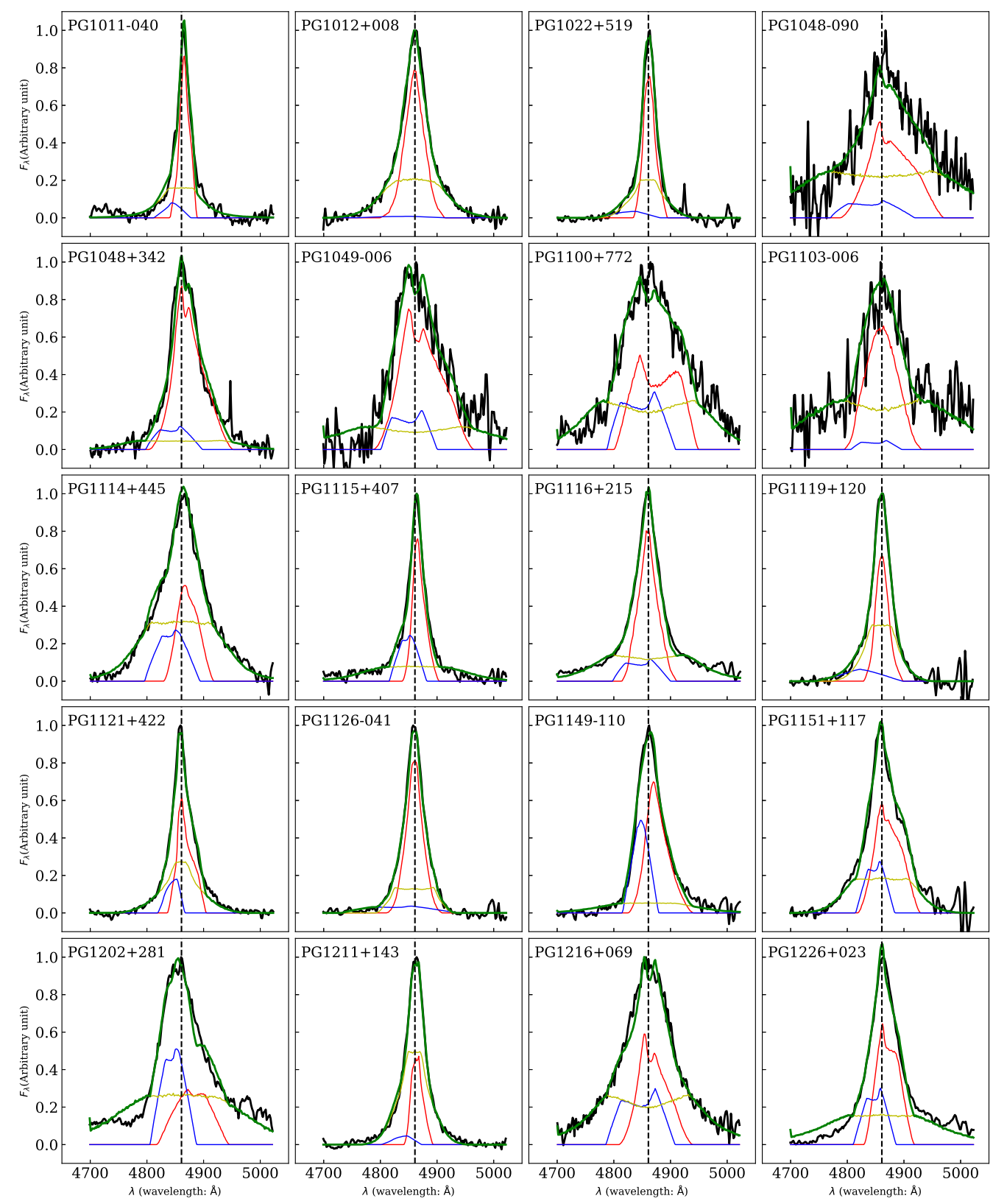

Supplementary Figure 1 Continued. 


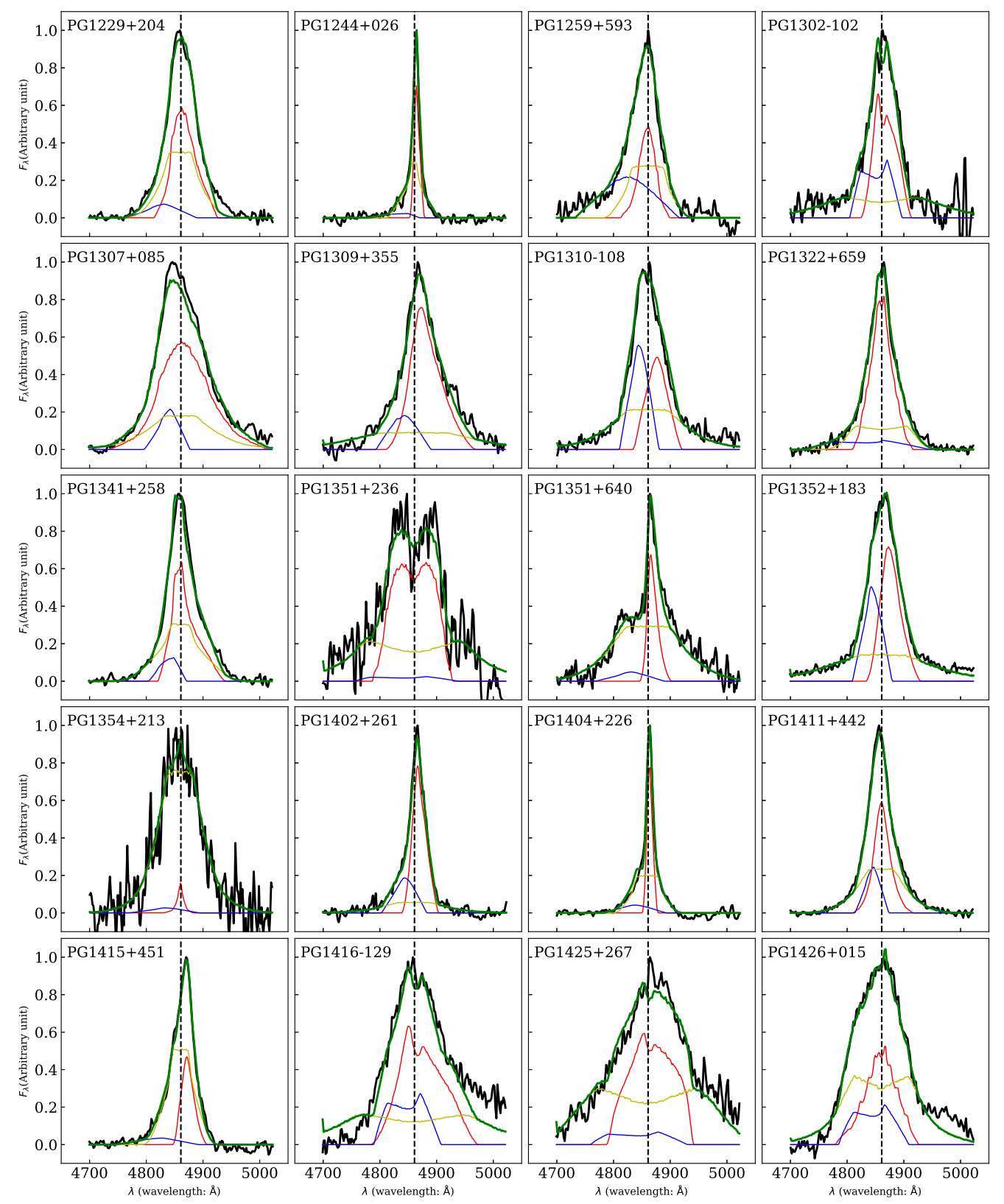

Supplementary Figure 1 Continued. 


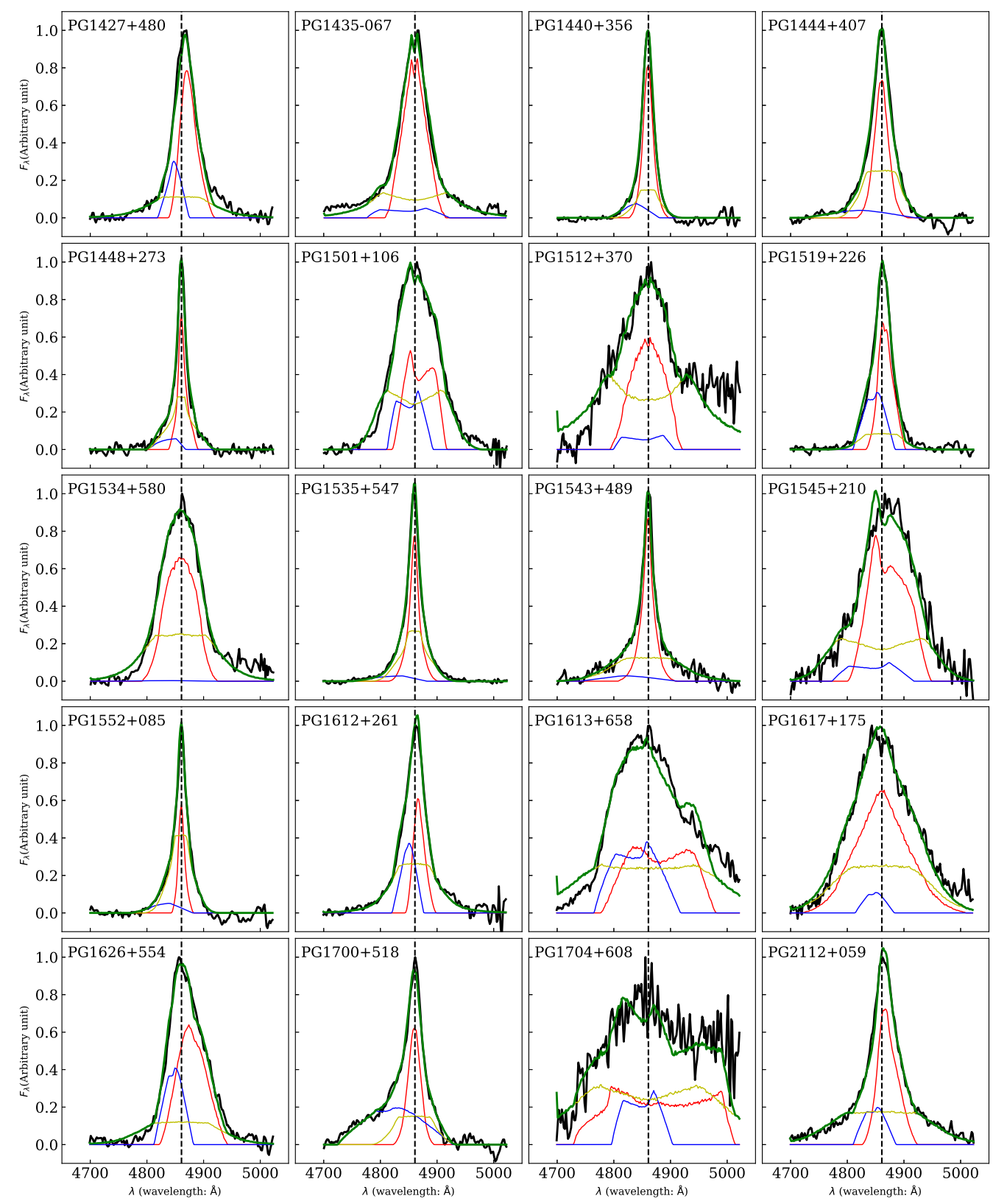

Supplementary Figure 1 Continued. 


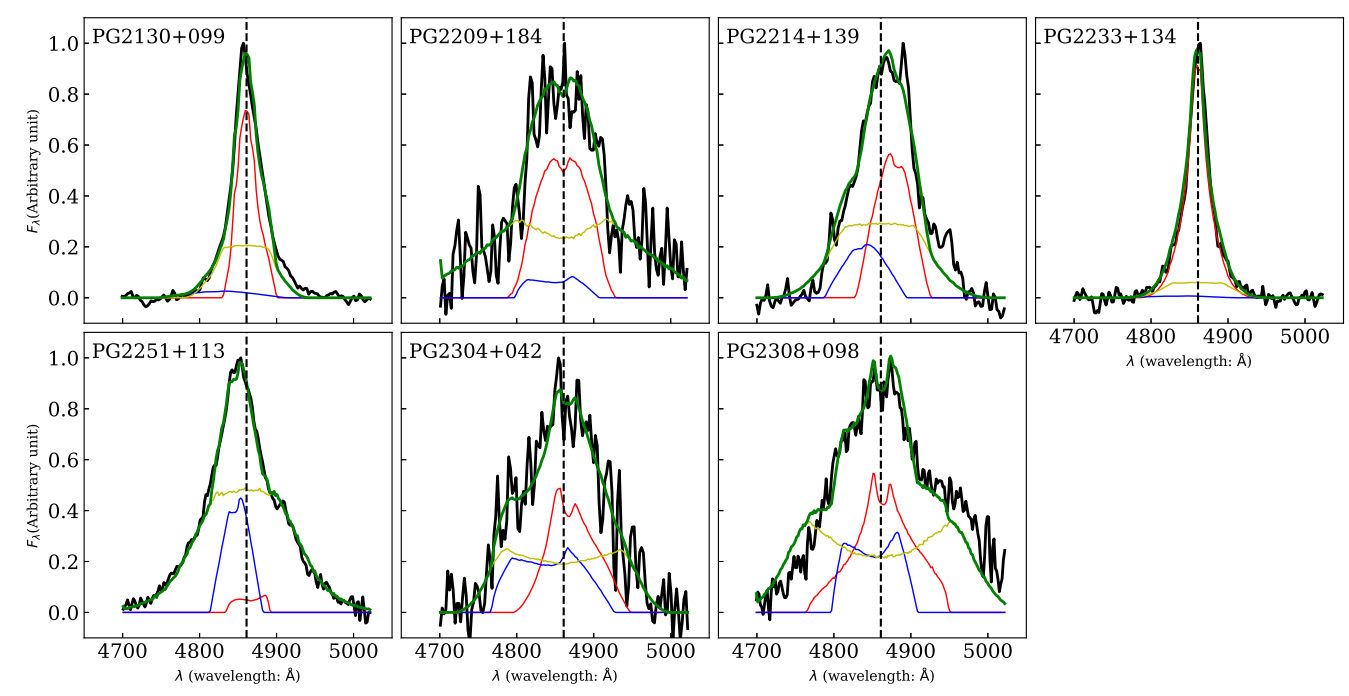

Supplementary Figure 1 Continued. 

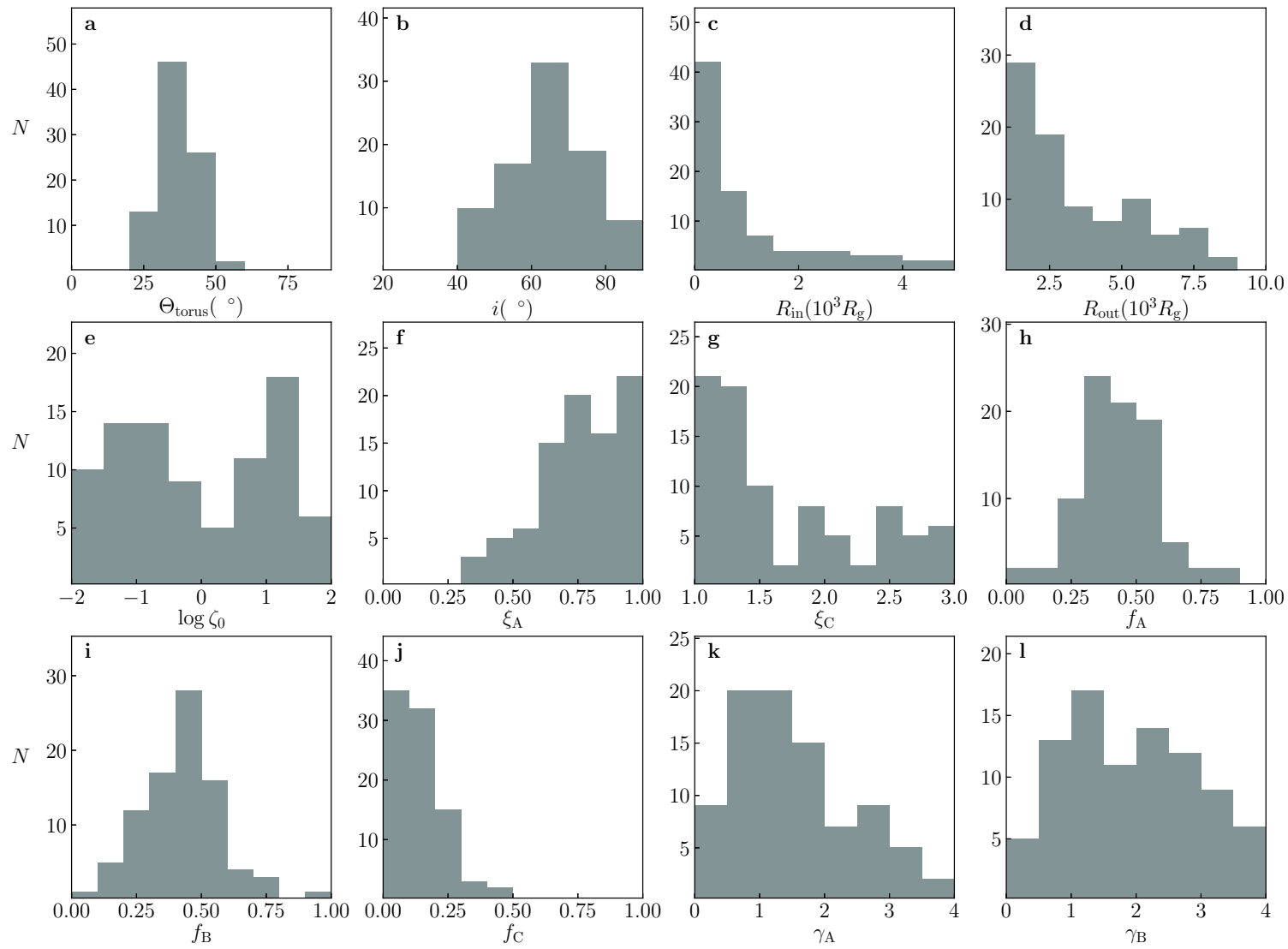

Supplementary Figure 2: Distributions of the parameters obtained by fitting $\mathbf{H} \beta$ profiles. Note $f_{\mathrm{C}}=1-\left(f_{\mathrm{A}}+f_{\mathrm{B}}\right)$,

the average fraction of type $\mathrm{C}$ clouds is $\left\langle f_{\mathrm{C}}\right\rangle \approx 0.1$ and $f_{\mathrm{C}} \ll\left(f_{\mathrm{B}}, f_{\mathrm{A}}\right)$ holds for most objects of the PG sample. There are a couple of objects with $\xi_{\mathrm{C}} \gtrsim 2$, potentially implying acceleration of type $\mathrm{C}$ clouds driven by radiation pressure, but this possibility needs to be explored by numerical simulations. 

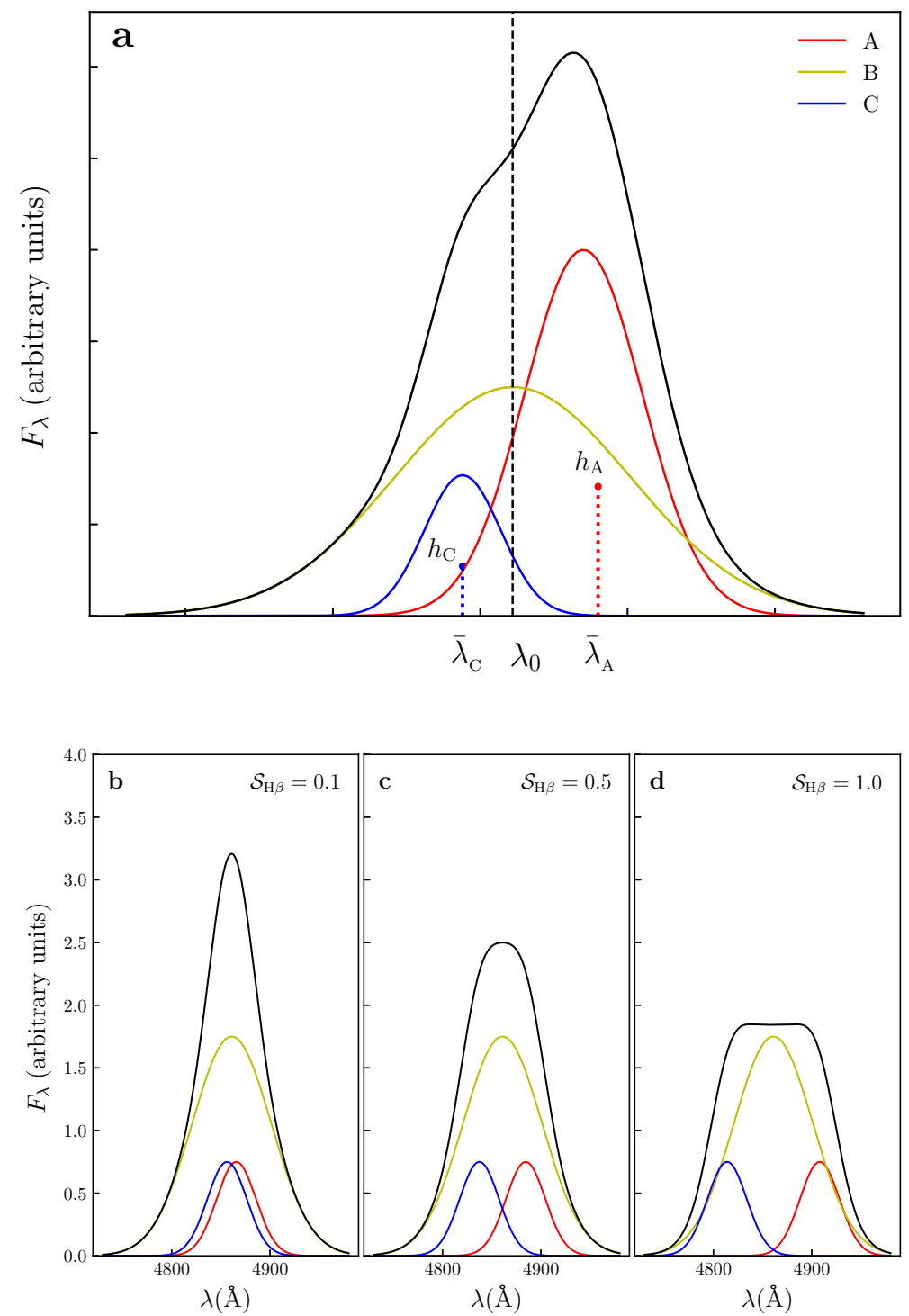

Supplementary Figure 3: Illustration of physical meanings of parameters describing profiles. Panel $a$ shows a cartoon of decomposed profiles for physical meanings of the three parameters defined by Equation (16). The barycentre wavelength and fluxes are obtained by including flux-weight. As the first order approximation, the two parameters of $(h, \bar{\lambda})$ can completely describe individual profiles of the decomposed components and hence provide reasonable asymmetries, shapes and shifts of the total spectra. Panels $b$ - $d$ illustrate the relationship between $\mathcal{S}_{\mathrm{H} \beta}$ and real shapes of profiles (from a triangular to boxy). 


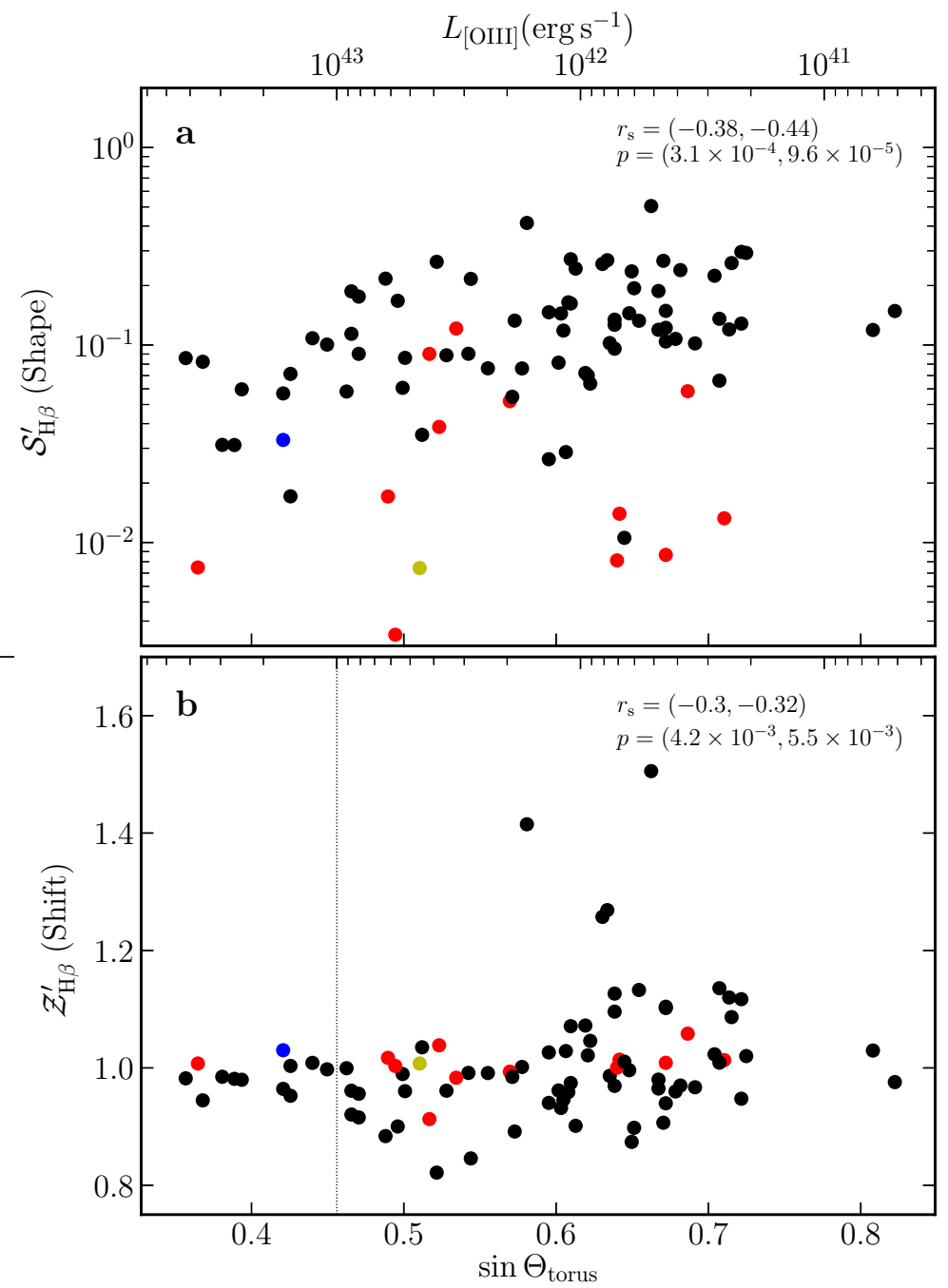

Supplementary Figure 4: Correlations between $\mathcal{S}_{\mathrm{H} \beta}^{\prime}$ and $\mathcal{Z}_{\mathrm{H} \beta}^{\prime}$ versus $L_{[\mathrm{OIII}]}$. Panel a shows a consistent correlation with panel $b$ in Figure 4 . There are four outliers with $\mathcal{Z}_{\mathrm{H} \beta}^{\prime} \gtrsim 1.2$ deviating from the correlation in panel $b$. For objects with $L_{[\mathrm{OIII}]} \gtrsim 10^{43} \mathrm{erg} \mathrm{s}^{-1}, \mathcal{Z}_{\mathrm{H} \beta}^{\prime} \approx 1$ remains, which have smaller torus angles $\left(\Theta_{\text {torus }} \lesssim 30^{\circ}\right)$. 


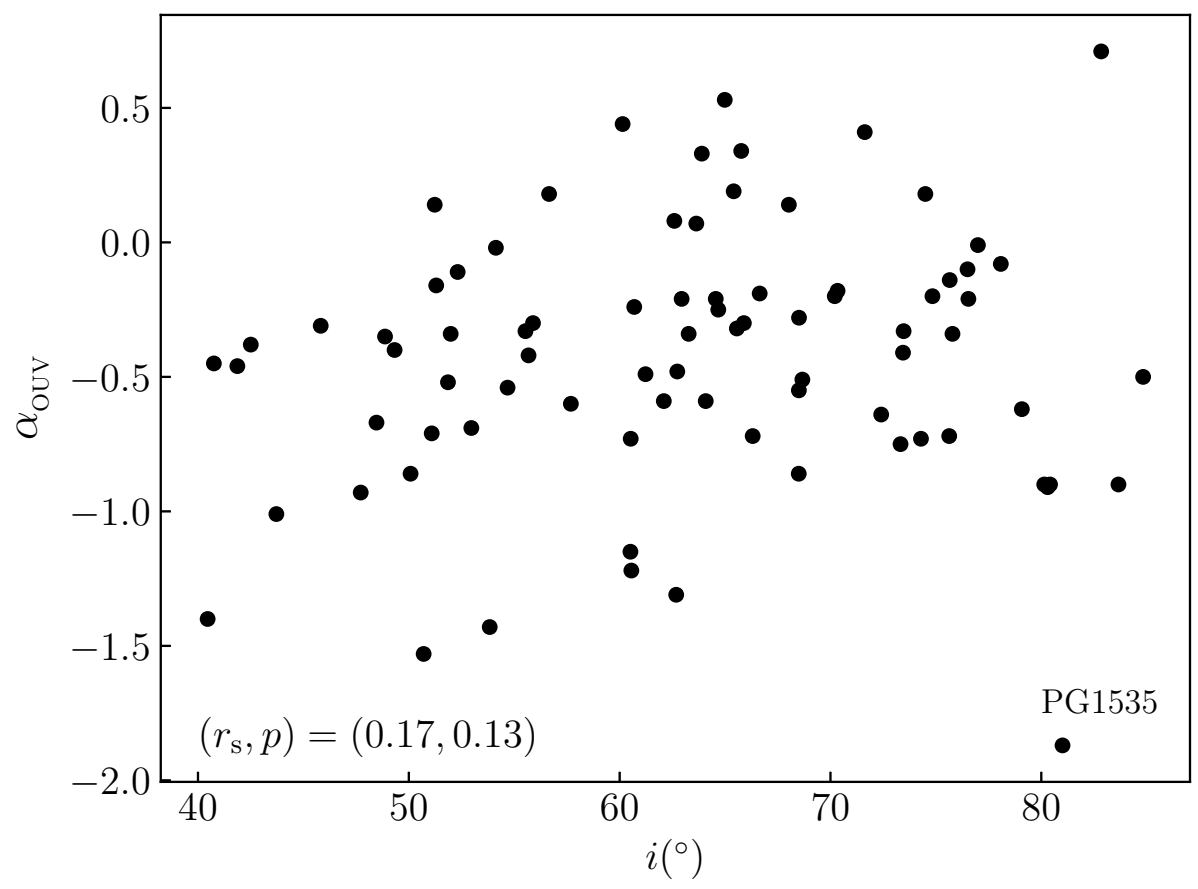

Supplementary Figure 5: A comparison of PG quasar inclinations from the present model with $\alpha_{\text {OUV }}$ as an orientation $^{2}$. This shows that they are consistent with each others. The Spearman coefficient and null-probability are indicated in the plot except for PG 1535. Error bars of $\alpha_{\mathrm{OUV}}$ are not given by Ref. ${ }^{2}$, and $i$ is given in Supplementary Table 1.

1. Reyes, R. et al. Space density of optically selected type 2 quasars. Astron. J. 136, 2373-2390 (2008).

2. Baskin, A. \& Laor, A. What controls the C IV line profile in active galactic nuclei? Mon. Not. R. Astron. Soc. 356, 1029-1044 (2005). 